\title{
Mathematical programming with multiple sets split monotone variational inclusion constraints
}

\author{
Zenn-Tsun Yu' ${ }^{1}$ Lai-Jiu Lin ${ }^{2 *}$ and Chih-Sheng Chuang ${ }^{3}$
}

\section{"Correspondence:}

maljlin@cc.ncue.edu.tw

${ }^{2}$ Department of Mathematics,

National Changhua University of

Education, Changhua, 50058,

Taiwan

Full list of author information is

available at the end of the article

\begin{abstract}
In this paper, we first study a hierarchical problem of Baillon's type, and we study a strong convergence theorem of this problem. For the special case of this convergence theorem, we obtain a strong convergence theorem for the ergodic theorem of Baillon's type. Our result of the ergodic theorem of Baillon's type improves and generalizes many existence theorems of this type of problem. Two numerical examples are given to demonstrate our results.

As applications of our convergence theorem of the hierarchical problem, we study the unique solution for the following problems: mathematical programming with multiply sets split variational inclusion and fixed point set constraints; mathematical programming with multiple sets split variational inequalities and fixed point set constraints; the variational inequality problem with a system of mixed type equilibria and fixed point set constraints; the variational inequality problem with multiple sets split system of mixed type equilibria and fixed point set constraints; mathematical programming with a system of mixed type equilibria and fixed point set constraints. We give iteration processes for these types of problems and establish the strong convergence for the unique solution of these problems. For our special case, our results can be reduced to the following problems: the unique minimal norm solution of the multiply sets split monotonic variational inclusion problems; the minimum norm solutions for the multiple sets split system of mixed type equilibria problem; the minimum norm solution of the system of mixed type equilibria problem. Our results will have many applications in diverse fields of science.
\end{abstract}

Keywords: hierarchical problems; split variational inclusion problems; fixed point problems; mathematical programming; minimum norm solution

\section{Introduction}

Let $C_{1}, C_{2}, \ldots, C_{m}$ be nonempty closed convex subsets of a Hilbert space $H_{1}$. The wellknown convex feasiblity problem (CFP) is to find $x^{*} \in H_{1}$ such that

$$
x^{*} \in C_{1} \cap C_{2} \cap \cdots \cap C_{m} .
$$

The split feasibility problem (SFP) is to find a point

$$
x^{*} \in C \text { such that } A x^{*} \in Q \text {, }
$$

O2014 Yu et al.; licensee Springer. This is an Open Access article distributed under the terms of the Creative Commons Attribution License (http://creativecommons.org/licenses/by/2.0), which permits unrestricted use, distribution, and reproduction in any medium, provided the original work is properly cited. 
where $C$ is a nonempty closed convex subset of a Hilbert space $H_{1}, Q$ is a nonempty closed convex subset of a Hilbert space $H_{2}$, and $A: H_{1} \rightarrow H_{2}$ is an operation. The split feasibility problem (SFP) in the finite dimensional Hilbert spaces was first introduced by Censor et al. [1] for modeling inverse problems which arise from phase retrievals and in medical image reconstruction. Since then, the convex feasibility problem and the split feasibility problem (SFP) has received much attention due to its applications in signal processing, image reconstruction, approximation theory, control theory, biomedical engineering, communications, and geophysics. For example, one can refer to [1-5] and related literature.

Let $C_{1}, C_{2}, \ldots, C_{m}$ be nonempty closed convex subsets of a Hilbert space $H_{1}$, let $Q_{1}, Q_{2}, \ldots, Q_{n}$ be nonempty closed convex sets $H_{2}$ and let $A_{1}, A_{2}, \ldots, A_{m}: H_{1} \rightarrow H_{2}$ be linear operator operators. The well-known multiple sets split feasibility problem studied by Censor et al. [6]. Xu [7] and Lopez et al. [8] (MSSFP) is to find $x^{*} \in H_{1}$ such that

$$
x^{*} \in C_{i} \text { such that } A_{i} x^{*} \in Q_{i} \quad \text { for all } i=1,2, \ldots, m .
$$

In 2011, Moudafi [9] introduced and studied the following split monotone variational inclusion (SMVI):

Find $\bar{x} \in H_{1}$ such that $\bar{x} \in\left(B_{1}+G_{1}\right)^{-1} 0$,

and

$$
\bar{y}=A \bar{x} \in H_{2} \text { such that } \bar{y} \in(B+G)^{-1} 0,
$$

where $H_{1}$ and $H_{2}$ are real Hilbert spaces, $A: H_{1} \rightarrow H_{2}$ is a bounded linear operator, $B_{1}$ : $H_{1} \rightarrow H_{1}$ and $B: H_{2} \rightarrow H_{2}$ are given operators, $G_{1}: H_{1} \multimap H_{1}$ and $G: H_{2} \multimap H_{2}$ are given multivalued mappings.

Moudafi [9] proved a weakly convergence theorem for the solution of the split monotone variational inclusion (SMVI) with an iteration process.

In 2011, Maruyama et al. [10] proved the following ergodic theorem of Baillon's type [11].

Theorem 1.1 [10] Let $C$ be a nonempty closed convex subset of a real Hilbert space $H$, $T: C \rightarrow C$ be a 2-generalized hybrid mapping with $\operatorname{Fix}(T) \neq \emptyset$ and let $P_{\mathrm{Fix}(T)}$ be the metric projection of $H_{1}$ onto $\operatorname{Fix}(T)$. Then, for any $x \in C$,

$$
S_{n} x:=\frac{1}{n} \sum_{k=0}^{n-1} T^{k} x
$$

converges weakly to an element $p$ of $\operatorname{Fix}(T)$, where $p=\lim _{n \rightarrow \infty} P_{\mathrm{Fix}(T)} T^{n} x$.

In this paper, we first study a hierarchical problem of Baillon's type, and we study a strong convergence theorem of this problem. For the special case of this convergence theorem, we obtain a strong convergence theorem for the ergodic theorem of Baillon's type. Our result of the ergodic theorem of Baillon's type improves and generalizes many existence theorems of this type of problem. Two numerical examples are given to demonstrate our results. 
As applications of our convergence theorem of the hierarchical problem, we study the unique solution for the following problems: mathematical programming with multiply sets split variational inclusion and a fixed point set constraints; mathematical programming with multiple sets split variational inequalities and fixed point set constraints; the variational inequality problem with a system of mixed type equilibria and fixed point set constraints; the variational inequality problem with multiple sets split system of mixed type equilibria and a fixed point set constraints; mathematical programming with system of mixed type equilibrium and a fixed point set constraints. We give iteration processes for these types of problems and establish the strong convergence for the unique solution of these problems. For the special case of our results, our results can be reduced to the following problems: the unique minimal norm solution of the multiply sets split monotonic variational inclusion problems; the minimum norm solutions for the multiple sets split system of mixed type equilibrium problem; the minimum norm solution of the system of the mixed type equilibria problem. Our results will have many applications in diverse fields of science.

\section{Preliminaries}

Throughout this paper, let $\mathbb{N}$ be the set of positive integers and let $\mathbb{R}$ be the set of real numbers, $H_{1}$ be a (real) Hilbert space with inner product $\langle\cdot, \cdot\rangle$ and norm $\|\cdot\|$, respectively, and $C$ be a nonempty closed convex subset of $H_{1}$. We denote the strongly convergence and the weak convergence of $\left\{x_{n}\right\}$ to $x \in H_{1}$ by $x_{n} \rightarrow x$ and $x_{n} \rightarrow x$, respectively.

Let $T: C \rightarrow H_{1}$ be a mapping, and let $\operatorname{Fix}(T):=\{x \in C: T x=x\}$ denote the set of fixed points of $T$. A mapping $T: C \rightarrow H_{1}$ is called

(i) a 2-generalized hybrid mapping [10] if there exist $\delta_{1}, \delta_{2}, \epsilon_{1}, \epsilon_{2} \in \mathbb{R}$ such that

$$
\begin{gathered}
\delta_{1}\left\|T^{2} x-T y\right\|^{2}+\delta_{2}\|T x-T y\|^{2}+\left(1-\delta_{1}-\delta_{2}\right)\|x-T y\|^{2} \\
\quad \leq \epsilon_{1}\left\|T^{2} x-y\right\|^{2}+\epsilon_{2}\|T x-y\|^{2}+\left(1-\epsilon_{1}-\epsilon_{2}\right)\|x-y\|^{2}
\end{gathered}
$$

for all $x, y \in C$.

We know that the class of 2-generalized hybrid mapping contains the classes of nonexpansive mappings, nonspreading mappings, and a $(\alpha, \beta)$-generalized hybrid [12] in a Hilbert space. We give an example for a 2-generalized hybrid mapping.

Example 2.1 [13] Let $T:[0,2] \rightarrow \mathbb{R}$ be defined as

$$
T x= \begin{cases}0 & \text { if } x \in[0,2) \\ 1 & \text { if } x=2\end{cases}
$$

Then $T$ is a 2-generalized hybrid mapping and $\operatorname{Fix}(T)=\{0\}$.

Proof Let $\epsilon_{1}=\epsilon_{2}=\frac{1}{4}, \delta_{1}=\delta_{2}=\frac{1}{2}$.

Case 1: If $x \in[0,2), y=2$, then $T x=T^{2} x=0, T y=1$ and $\|x-T y\| \leq 1$. We know that

$$
\begin{aligned}
\delta_{1} \| & T^{2} x-T y\left\|^{2}+\delta_{2}\right\| T x-T y\left\|^{2}+\left(1-\delta_{1}-\delta_{2}\right)\right\| x-T y \|^{2} \\
& =\delta_{1}+\delta_{2}+\left(1-\delta_{1}-\delta_{2}\right)\|x-T y\|^{2} \\
& \leq \delta_{1}+\delta_{2}+\left(1-\delta_{1}-\delta_{2}\right)=1
\end{aligned}
$$


and

$$
\begin{aligned}
\epsilon_{1} & \left\|T^{2} x-y\right\|^{2}+\epsilon_{2}\|T x-y\|^{2}+\left(1-\epsilon_{1}-\epsilon_{2}\right)\|x-y\|^{2} \\
& =4 \epsilon_{1}+4 \epsilon_{2}+\left(1-\epsilon_{1}-\epsilon_{2}\right)\|x-2\|^{2} \\
& \geq 4 \epsilon_{1}+4 \epsilon_{2} \geq 1+1=2 .
\end{aligned}
$$

Therefore,

$$
\begin{gathered}
\delta_{1}\left\|T^{2} x-T y\right\|^{2}+\delta_{2}\|T x-T y\|^{2}+\left(1-\delta_{1}-\delta_{2}\right)\|x-T y\|^{2} \\
\leq \epsilon_{1}\left\|T^{2} x-y\right\|^{2}+\epsilon_{2}\|T x-y\|^{2}+\left(1-\epsilon_{1}-\epsilon_{2}\right)\|x-y\|^{2} .
\end{gathered}
$$

Case 2: If $x \in[0,2), y \in[0,2)$, then $T x=T^{2} x=0, T y=T^{2} y=0$. We know that

$$
\begin{aligned}
\delta_{1} \| & T^{2} x-T y\left\|^{2}+\delta_{2}\right\| T x-T y\left\|^{2}+\left(1-\delta_{1}-\delta_{2}\right)\right\| x-T y \|^{2} \\
& =\left(1-\delta_{1}-\delta_{2}\right) x^{2} \\
& =0 \\
& \leq \epsilon_{1}\left\|T^{2} x-y\right\|^{2}+\epsilon_{2}\|T x-y\|^{2}+\left(1-\epsilon_{1}-\epsilon_{2}\right)\|x-y\|^{2} .
\end{aligned}
$$

Case 3: If $x=y=2$, then $T x=1, T^{2} x=0, T y=1, T^{2} y=0$. We know that

$$
\begin{aligned}
\delta_{1} \| & T^{2} x-T y\left\|^{2}+\delta_{2}\right\| T x-T y\left\|^{2}+\left(1-\delta_{1}-\delta_{2}\right)\right\| x-T y \|^{2} \\
& =\delta_{1}+\left(1-\delta_{1}-\delta_{2}\right) \\
& =\left(1-\delta_{2}\right)=\frac{1}{2}
\end{aligned}
$$

and

$$
\begin{aligned}
\epsilon_{1} \| & T^{2} x-y\left\|^{2}+\epsilon_{2}\right\| T x-y\left\|^{2}+\left(1-\epsilon_{1}-\epsilon_{2}\right)\right\| x-y \|^{2} \\
& =4 \epsilon_{1}+\epsilon_{2} \geq \frac{5}{4} .
\end{aligned}
$$

Therefore,

$$
\begin{aligned}
& \delta_{1}\left\|T^{2} x-T y\right\|^{2}+\delta_{2}\|T x-T y\|^{2}+\left(1-\delta_{1}-\delta_{2}\right)\|x-T y\|^{2} \\
& \leq \epsilon_{1}\left\|T^{2} x-y\right\|^{2}+\epsilon_{2}\|T x-y\|^{2}+\left(1-\epsilon_{1}-\epsilon_{2}\right)\|x-y\|^{2} .
\end{aligned}
$$

By the above case, we know that $T$ is a 2-generalized hybrid.

A mapping $V: H_{1} \rightarrow H_{1}$ is called

(i) strongly monotone if there exists $\bar{\gamma}>0$ such that $\langle x-y, V x-V y\rangle \geq \bar{\gamma}\|x-y\|^{2}$ for all $x, y \in H_{1}$

(ii) $\alpha$-inverse-strongly monotone if $\langle x-y, V x-V y\rangle \geq \alpha\|V x-V y\|^{2}$ for all $x, y \in H_{1}$ and $\alpha>0$. 
We also know that if $V$ is a $\alpha$-inverse-strongly monotone mapping and $0<\lambda \leq 2 \alpha$, then $I-\lambda V: C \rightarrow H_{1}$ is nonexpansive.

Let $G: H_{1} \multimap H_{1}$ be a multivalued mapping. The effective domain of $G$ is denoted by $D(G)$, that is, $D(G)=\left\{x \in H_{1}: G x \neq \emptyset\right\}$.

Then $G: H_{1} \multimap H_{1}$ is called

(i) a monotone operator on $H_{1}$ if $\langle x-y, u-v\rangle \geq 0$ for all $x, y \in D(G), u \in G x$, and $v \in G y$

(ii) a maximal monotone operator on $H_{1}$ if $G$ is a monotone operator on $H_{1}$ and its graph is not properly contained in the graph of any other monotone operator on $H_{1}$.

Lemma 2.1 [14] Let $C$ be a nonempty closed convex subset of a real Hilbert space $H_{1}$. Let $T$ be a nonexpansive mapping of $C$ into itself, and let $\left\{x_{n}\right\}$ be a sequence in C. If $x_{n} \rightarrow w$ and $\lim _{n \rightarrow \infty}\left\|x_{n}-T x_{n}\right\|=0$, then $T w=w$.

In 2012, Hojo et al. [15] also gave an example for a 2-generalized hybrid mapping which is not a generalized hybrid mapping with $\operatorname{Fix}(T)=\{(0,0)\}$. We shall prove that this example for a 2-generalized hybrid mapping does not satisfy the demiclosed property as in Lemma 2.1 .

Example 2.2 Let $A=\left\{x \in \mathbb{R}^{2}:\|x\| \leq 1\right\}$ and $T A \rightarrow \mathbb{R}^{2}$ be defined as

$$
T x= \begin{cases}(0,0) & \text { if } x \in A, \\ \frac{x}{\|x\|} & \text { if } x \in \mathbb{R}^{2} / A .\end{cases}
$$

Hojo et al. [15] showed that $T$ is a 2-generalized hybrid mapping, but $T$ is not a generalized hybrid mapping. Note that $T$ does not have the demiclosed property. Indeed, there exists a sequence $\left\{x_{n}\right\} \in A$ such that $x_{n} \rightarrow w$ and $\lim _{n \rightarrow \infty}\left\|x_{n}-T x_{n}\right\|=0$, but $w$ in $\mathbb{R}^{2} / \operatorname{Fix}(T)=\mathbb{R}^{2} /\{(0,0)\}$.

Proof Let $r_{n}=1+\frac{1}{n}, x_{n}=\left(r_{n} \cos \theta, r_{n} \sin \theta\right)$ for all $n \in \mathbb{N}$, then $x_{n} \rightarrow(\cos \theta, \sin \theta)$ and $T x_{n}=$ $(\cos \theta, \sin \theta)$. We also have $\left\|T x_{n}-x_{n}\right\|=\left\|\left(\left(r_{n}-1\right) \cos \theta,\left(r_{n}-1\right) \sin \theta\right)\right\|=r_{n}-1 \rightarrow 0$, but $(\cos \theta, \sin \theta) \neq(0,0)$.

Lemma 2.2 [16] Let $V: H_{1} \rightarrow H_{1}$ be a $\bar{\gamma}$-strongly monotone and L-Lipschitzian continuous operator with $\bar{\gamma}>0$ and $L>0$. Let $\theta \in H_{1}$, and $V_{1}: H_{1} \rightarrow H_{1}$ such that $V_{1} x=V x-\theta$. Then $V_{1}$ is a $\bar{\gamma}$-strongly monotone and L-Lipschitzian continuous mapping. Furthermore, there is a unique fixed point $z_{0}$ in $C$ satisfying $z_{0}=P_{C}\left(z_{0}-V z_{0}+\theta\right)$. This point $z_{0} \in C$ is also a unique solution of the hierarchical variational inequality $\left\langle V z_{0}-\theta, q-z_{0}\right\rangle \geq 0$, for all $q \in C$.

Let $C$ be a nonempty subset of a real Hilbert space $H_{1}$. Then $T: C \rightarrow H_{1}$ is a firmly nonexpansive mapping if $\|T x-T y\|^{2} \leq\|x-y\|^{2}-\|(I-T) x-(I-T) y\|^{2}$ for every $x, y \in C$, that is, $\|T x-T y\|^{2} \leq\langle x-y, T x-T y\rangle$ for every $x, y \in C$.

Lemma 2.3 [17] Let $G_{1}$ be a maximal monotone mapping on $H_{1}$. Let $J_{r}^{G_{1}}$ be the resolvent of $G_{1}$ defined by $J_{r}^{G_{1}}=\left(I+r G_{1}\right)^{-1}$ for each $r>0$. Then the following holds:

$$
\frac{|s-t|}{s}\left\langle J_{s}^{G_{1}} x-J_{t}^{G_{1}} x, J_{s}^{G_{1}} x-x\right\rangle \geq\left\|J_{s}^{G_{1}} x-J_{t}^{G_{1}} x\right\|^{2}
$$


for all $s, t>0$ and $x \in H_{1}$. In particular,

$$
\left\|J_{s}^{G_{1}} x-J_{t}^{G_{1}} x\right\| \leq \frac{|s-t|}{s}\left\|J_{s}^{G_{1}} x-x\right\|
$$

for all $s, t>0$ and $x \in H_{1}$.

A mapping $T: H_{1} \rightarrow H_{1}$ is said to be averaged if $T=(1-\alpha) I+\alpha S$, where $\alpha \in(0,1)$ and $S: H_{1} \rightarrow H_{1}$ is nonexpansive. In this case, we also say that $T$ is $\alpha$-averaged. A firmly nonexpansive mapping is $\frac{1}{2}$-averaged.

Lemma 2.4 $([7,18])$ Let $C$ be a nonempty closed convex subset of a real Hilbert space $H$, and let $T: C \rightarrow C$ be a mapping. Then the following are satisfied:

(i) $T$ is nonexpansive if and only if the complement $(I-T)$ is 1/2-ism.

(ii) If $S$ is $v$-ism, then for $\gamma>0, \gamma S$ is $v / \gamma$-ism.

(iii) $S$ is averaged if and only if the complement $I-S$ is $v$-ism for some $v>1 / 2$.

(iv) If $S$ and $T$ are both averaged, then the product (composite) $S T$ is averaged.

(v) If the mappings $\left\{T_{i}\right\}_{i=1}^{n}$ are averaged and have a common fixed point, then $\bigcap_{i=1}^{n} \operatorname{Fix}\left(T_{i}\right)=\operatorname{Fix}\left(T_{1} \cdots T_{n}\right)$.

Lemma 2.5[19] Let $\left\{a_{n}\right\}$ be a sequence of real numbers such that there exists a subsequence $\left\{n_{i}\right\}$ of $\{n\}$ such that $a_{n_{i}}<a_{n_{i}+1}$ for all $i \in \mathbb{N}$. Then there exists a nondecreasing sequence $\left\{m_{k}\right\} \subseteq \mathbb{N}$ such that $m_{k} \rightarrow \infty$ and the following properties are satisfied by all (sufficiently large) numbers $k \in \mathbb{N}$ :

$$
a_{m_{k}} \leq a_{m_{k}+1} \text { and } a_{k} \leq a_{m_{k}+1} \text {. }
$$

In fact, $m_{k}=\max \left\{j \leq k: a_{j}<a_{j+1}\right\}$.

Lemma 2.6 [20] Let $\left\{a_{n}\right\}_{n \in \mathbb{N}}$ be a sequence of nonnegative real numbers, $\left\{\alpha_{n}\right\}$ a sequence of real numbers in $[0,1]$ with $\sum_{n=1}^{\infty} \alpha_{n}=\infty,\left\{u_{n}\right\}$ a sequence of nonnegative real numbers with $\sum_{n=1}^{\infty} u_{n}<\infty,\left\{t_{n}\right\}$ a sequence of real numbers with lim sup $t_{n} \leq 0$. Suppose that $a_{n+1} \leq$ $\left(1-\alpha_{n}\right) a_{n}+\alpha_{n} t_{n}+u_{n}$ for each $n \in \mathbb{N}$. Then $\lim _{n \rightarrow \infty} a_{n}=0$.

\section{Convergence theorems of hierarchical problems}

Let $H_{1}$ be a real Hilbert space and let $C$ be a nonempty closed convex subset of $H_{1}$. For each $i=1,2$, and $\kappa_{i}>0$, let $F_{i}$ be a $\kappa_{i}$-inverse-strongly monotone mapping of $C$ into $H_{1}$. For each $i=1,2$, let $G_{i}$ be a maximal monotone mapping on $H_{1}$ such that the domain of $G_{i}$ is included in $C$ and define the set $G_{i}^{-1} 0$ as $G_{i}^{-1} 0=\left\{x \in H_{1}: 0 \in G_{i} x\right\}$. Let $J_{\lambda_{n}}^{G_{1}}=\left(I+\lambda_{n} G_{1}\right)^{-1}$ and $J_{r_{n}}^{G_{2}}=\left(I+r_{n} G_{2}\right)^{-1}$ for each $n \in \mathbb{N}, \lambda_{n}>0$ and $r_{n}>0$. Let $\left\{\theta_{n}\right\} \subset H_{1}$ be a sequence. Let $V$ be a $\bar{\gamma}$-strongly monotone and $L$-Lipschitzian continuous operator with $\bar{\gamma}>0$ and $L>0$. Throughout this paper, we use these notations and assumptions unless specified otherwise.

The following strong convergence theorem for hierarchical problems is one of our main results in this paper. 
Theorem 3.1 Let $T: C \rightarrow H_{1}$ be a 2-generalized hybrid mapping with $\operatorname{Fix}(T) \cap\left(F_{1}+\right.$ $\left.G_{1}\right)^{-1} 0 \cap\left(F_{2}+G_{2}\right)^{-1} 0 \neq \emptyset$. Take $\mu \in \mathbb{R}$ as follows:

$$
0<\mu<\frac{2 \bar{\gamma}}{L^{2}} .
$$

Let $\left\{x_{n}\right\} \subset H_{1}$ be defined by

$$
\left\{\begin{array}{l}
x_{1} \in C \text { chosen arbitrarily, } \\
y_{n}=J_{\lambda_{n}}^{G_{1}}\left(I-\lambda_{n} F_{1}\right) J_{r_{n}}^{G_{2}}\left(I-r_{n} F_{2}\right) x_{n}, \\
s_{n}=\frac{1}{n} \sum_{k=0}^{n-1} T^{k} y_{n} \\
x_{n+1}=\alpha_{n} x_{n}+\left(1-\alpha_{n}\right)\left(\beta_{n} \theta_{n}+\left(I-\beta_{n} V\right) s_{n}\right)
\end{array}\right.
$$

for each $n \in \mathbb{N},\left\{\lambda_{n}\right\} \subset(0, \infty),\left\{\alpha_{n}\right\} \subset(0,1),\left\{\beta_{n}\right\} \subset(0,1)$, and $\left\{r_{n}\right\} \subset(0, \infty)$. Assume that:

(i) $0<\liminf _{n \rightarrow \infty} \alpha_{n} \leq \limsup _{n \rightarrow \infty} \alpha_{n}<1$;

(ii) $\lim _{n \rightarrow \infty} \beta_{n}=0$, and $\sum_{n=1}^{\infty} \beta_{n}=\infty$;

(iii) $0<a_{1} \leq \lambda_{n} \leq b_{1}<2 \kappa_{1}$, and $0<a_{2} \leq r_{n} \leq b_{2}<2 \kappa_{2}$;

(iv) $\lim _{n \rightarrow \infty} \theta_{n}=\theta$ for some $\theta \in H_{1}$.

Then $\lim _{n \rightarrow \infty} x_{n}=\bar{x}$, where $\bar{x}=P_{\mathrm{Fix}(T) \cap\left(F_{1}+G_{1}\right)^{-1} 0 \cap\left(F_{2}+G_{2}\right)^{-1} 0}(\bar{x}-V \bar{x}+\theta)$. This point $\bar{x}$ is also a unique solution of the hierarchical variational inequality:

$$
\langle V \bar{x}-\theta, q-\bar{x}\rangle \geq 0, \quad \forall q \in \operatorname{Fix}(T) \cap\left(F_{1}+G_{1}\right)^{-1} 0 \cap\left(F_{2}+G_{2}\right)^{-1} 0 .
$$

Proof Take any $\bar{x} \in \operatorname{Fix}(T) \cap\left(F_{1}+G_{1}\right)^{-1} 0 \cap\left(F_{2}+G_{2}\right)^{-1} 0$ and let $\bar{x}$ be fixed. Then we have $\bar{x}=J_{\lambda_{n}}^{G_{1}}\left(I-\lambda_{n} F_{1}\right) \bar{x}$ and $\bar{x}=J_{r_{n}}^{G_{2}}\left(I-r_{n} F_{2}\right) \bar{x}$. Let $u_{n}=J_{r_{n}}^{G_{2}}\left(I-r_{n} F_{2}\right) x_{n}$. For each $n \in \mathbb{N}$, by the same argument as the proof of Theorem 3.1 [16], we have

$$
\left\|u_{n}-\bar{x}\right\|^{2} \leq\left\|x_{n}-\bar{x}\right\|^{2}-r_{n}\left(2 \kappa_{2}-r_{n}\right)\left\|F_{2} x_{n}-F_{2} \bar{x}\right\|^{2} \leq\left\|x_{n}-\bar{x}\right\|^{2}
$$

and

$$
\left\|y_{n}-\bar{x}\right\|^{2} \leq\left\|u_{n}-\bar{x}\right\|^{2}-\lambda_{n}\left(2 \kappa_{1}-\lambda_{n}\right)\left\|F_{1} u_{n}-F_{1} \bar{x}\right\|^{2} \leq\left\|x_{n}-\bar{x}\right\|^{2} .
$$

By equations (3) and (4), we have

$$
\left\|y_{n}-\bar{x}\right\| \leq\left\|u_{n}-\bar{x}\right\| \leq\left\|x_{n}-\bar{x}\right\| .
$$

Since $T$ is a 2 -generalized hybrid mapping with $\operatorname{Fix}(T) \neq \emptyset$, we know that $T$ is a quasinonexpansive, and

$$
\begin{aligned}
\left\|s_{n}-\bar{x}\right\| & =\left\|\frac{1}{n} \sum_{k=0}^{n-1} T^{k} y_{n}-\bar{x}\right\| \leq \frac{1}{n} \sum_{k=0}^{n-1}\left\|T^{k} y_{n}-\bar{x}\right\| \\
& \leq\left\|y_{n}-\bar{x}\right\| \leq\left\|u_{n}-\bar{x}\right\| \leq\left\|x_{n}-\bar{x}\right\| .
\end{aligned}
$$

By the same argument as in the proof of Theorem 3.1 [16], we find that the sequence $\left\{x_{n}\right\}$ is bounded. Furthermore, $\left\{u_{n}\right\},\left\{z_{n}\right\},\left\{y_{n}\right\}$, and $\left\{s_{n}\right\}$ are bounded. We also have

$$
\left\|x_{n+1}-x_{n}\right\|^{2} \leq\left(1-\alpha_{n}\right)^{2}\left[\beta_{n}^{2}\left\|\theta_{n}-V s_{n}\right\|^{2}+\left\|s_{n}-x_{n}\right\|^{2}+2 \beta_{n}\left\|\theta_{n}-V s_{n}\right\|\left\|s_{n}-x_{n}\right\|\right]
$$


and

$$
\begin{aligned}
& \left\|x_{n+1}-\bar{x}\right\|^{2}-\left\|x_{n}-\bar{x}\right\|^{2}+\left(1-\alpha_{n}\right) \alpha_{n}\left\|s_{n}-x_{n}\right\|^{2} \\
& \leq 2\left(1-\alpha_{n}\right) \beta_{n}\left\langle\theta_{n}, x_{n}-\bar{x}\right\rangle-2\left(1-\alpha_{n}\right) \beta_{n}\left\langle V s_{n}, x_{n}-\bar{x}\right\rangle \\
& \quad+\left(1-\alpha_{n}\right)^{2}\left[\beta_{n}^{2}\left\|\theta_{n}-V s_{n}\right\|^{2}+2 \beta_{n}\left\|\theta_{n}-V s_{n}\right\|\left\|s_{n}-x_{n}\right\|\right] .
\end{aligned}
$$

We will divide the proof into two cases.

Case 1: there exists a natural number $N$ such that $\left\|x_{n+1}-\bar{x}\right\| \leq\left\|x_{n}-\bar{x}\right\|$ for each $n \geq N$. Therefore, $\lim _{n \rightarrow \infty}\left\|x_{n}-\bar{x}\right\|$ exists. Hence, it follows from equation (7), (i), and (ii) that

$$
\lim _{n \rightarrow \infty}\left\|s_{n}-x_{n}\right\|=0
$$

By equations (6), (8), (i), and (ii), we have

$$
\lim _{n \rightarrow \infty}\left\|x_{n+1}-x_{n}\right\|=0
$$

We also have

$$
\left\|z_{n}-s_{n}\right\| \leq\left\|\beta_{n} \theta_{n}+\left(1-\beta_{n} V\right) s_{n}-s_{n}\right\| \leq \beta_{n}\left\|\theta_{n}-V s_{n}\right\| .
$$

By equation (10), (iv), and (ii) we have

$$
\lim _{n \rightarrow \infty}\left\|z_{n}-s_{n}\right\|=0
$$

By equations (8) and (11),

$$
\lim _{n \rightarrow \infty}\left\|z_{n}-x_{n}\right\|=0
$$

By the same argument as in the proof of Theorem 3.1 [16], we have

$$
\lim _{n \rightarrow \infty}\left\|u_{n}-x_{n}\right\|=0
$$

and

$$
\lim _{n \rightarrow \infty}\left\|y_{n}-u_{n}\right\|=0
$$

Since $\operatorname{Fix}(T) \cap\left(F_{1}+G_{1}\right)^{-1} 0 \cap\left(F_{2}+G_{2}\right)^{-1} 0$ is a nonempty closed convex subset of $H_{1}$, by Lemma 2.2, we can take $\bar{x}_{0} \in \operatorname{Fix}(T) \cap\left(F_{1}+G_{1}\right)^{-1} 0 \cap\left(F_{2}+G_{2}\right)^{-1} 0$ such that

$$
\bar{x}_{0}=P_{\mathrm{Fix}(T) \cap\left(F_{1}+G_{1}\right)^{-1} 0 \cap\left(F_{2}+G_{2}\right)^{-1} 0}\left(\bar{x}_{0}-V \bar{x}_{0}+\theta\right) .
$$

This point $\bar{x}_{0}$ is also a unique solution of the hierarchical variational inequality:

$$
\left\langle V \bar{x}_{0}-\theta, q-\bar{x}_{0}\right\rangle \geq 0, \quad \forall q \in \operatorname{Fix}(T) \cap\left(F_{1}+G_{1}\right)^{-1} 0 \cap\left(F_{2}+G_{2}\right)^{-1} 0 .
$$


We shall show that

$$
\limsup _{n \rightarrow \infty}\left\langle V \bar{x}_{0}-\theta, z_{n}-\bar{x}_{0}\right\rangle \geq 0
$$

Without loss of generality, there exists a subsequence $\left\{z_{n_{k}}\right\}$ of $\left\{z_{n}\right\}$ such that

$$
z_{n_{k}} \rightarrow w
$$

for some $w \in H_{1}$ and

$$
\limsup _{n \rightarrow \infty}\left\langle V \bar{x}_{0}-\theta, z_{n}-\bar{x}_{0}\right\rangle=\lim _{k \rightarrow \infty}\left\langle V \bar{x}_{0}-\theta, z_{n_{k}}-\bar{x}_{0}\right\rangle .
$$

By equations (12) and (13), we have

$$
\lim _{n \rightarrow \infty}\left\|u_{n}-z_{n}\right\|=0
$$

and $u_{n_{k}} \rightarrow w$. On the other hand, since $0<a_{1} \leq \lambda_{n} \leq b_{1}<2 \kappa_{1}$, there exists a subsequence $\left\{\lambda_{n_{k_{j}}}\right\}$ of $\left\{\lambda_{n_{k}}\right\}$ such that $\left\{\lambda_{n_{k_{j}}}\right\}$ converges to a number $\bar{\lambda} \in\left[a_{1}, b_{1}\right]$. By equation (14) and Lemma 2.3, we have

$$
\begin{aligned}
& \left\|u_{n_{k_{j}}}-J_{\bar{\lambda}}^{G}\left(I-\bar{\lambda} F_{1}\right) u_{n_{k_{j}}}\right\| \\
& \leq\left\|u_{n_{k_{j}}}-J_{\lambda_{n_{k_{j}}}}^{G_{1}}\left(I-\lambda_{n_{k_{j}}} F_{1}\right) u_{n_{k_{j}}}\right\|+\left\|J_{\lambda_{n_{k_{j}}}}^{G_{1}}\left(I-\bar{\lambda} F_{1}\right) u_{n_{k_{j}}}-J_{\bar{\lambda}}^{G_{1}}\left(I-\bar{\lambda} F_{1}\right) u_{n_{k_{j}}}\right\| \\
& \quad+\left\|J_{\lambda_{n_{k_{j}}}}^{G_{1}}\left(I-\lambda_{n_{k_{j}}} F_{1}\right) u_{n_{k_{j}}}-J_{\lambda_{n_{k_{j}}}}^{G_{1}}\left(I-\bar{\lambda} F_{1}\right) u_{n_{k_{j}}}\right\| \\
& \leq\left\|u_{n_{k_{j}}}-y_{n_{k_{j}}}\right\|+\left|\lambda_{n_{k_{j}}}-\bar{\lambda}\right|\left\|F_{1} u_{n_{k_{j}}}\right\| \\
& \quad+\frac{\left|\lambda_{n_{k_{j}}}-\bar{\lambda}\right|}{\bar{\lambda}}\left\|J_{\bar{\lambda}}^{G}\left(I-\bar{\lambda} F_{1}\right) u_{n_{k_{j}}}-\left(I-\bar{\lambda} F_{1}\right) u_{n_{k_{j}}}\right\| \rightarrow 0 .
\end{aligned}
$$

By equation (18), $u_{n_{k_{j}}} \rightarrow w$, and Lemma 2.1, $w \in \operatorname{Fix}\left(J_{\bar{\lambda}}^{G_{1}}(I-\bar{\lambda} F)\right)=(F+B)^{-1} 0$.

Since $0<a_{2} \leq r_{n} \leq b_{2}<2 \kappa_{2}$, there exists a subsequence $\left\{r_{n_{k_{j}}}\right\}$ of $\left\{r_{n_{k}}\right\}$ such that $\left\{r_{n_{k_{j}}}\right\}$ converges to a number $\bar{r} \in\left[a_{2}, b_{2}\right]$. By the same argument as for equation (18), we have

$$
\left\|x_{n_{k_{j}}}-J_{\bar{r}}^{G_{2}}\left(I-\bar{r} F_{2}\right) x_{n_{k_{j}}}\right\| \rightarrow 0 .
$$

By equation (13) and $u_{n_{k}} \rightarrow w$, we have $x_{n_{k}} \rightarrow w$.

By equation (19), $x_{n_{k}} \rightarrow w$ and Lemma 2.1, we have $w \in \operatorname{Fix}\left(J_{\bar{r}}^{G_{2}}\left(I-\bar{r} F_{2}\right)\right)=\left(F_{2}+G_{2}\right)^{-1} 0$. Since $T$ is a 2-generalized hybrid mapping, there exist $\delta_{1}, \delta_{2}, \epsilon_{1}, \epsilon_{2} \in \mathbb{R}$ such that

$$
\begin{gathered}
\delta_{1}\left\|T^{2} x-T y\right\|^{2}+\delta_{2}\|T x-T y\|^{2}+\left(1-\delta_{1}-\delta_{2}\right)\|x-T y\|^{2} \\
\quad \leq \epsilon_{1}\left\|T^{2} x-y\right\|^{2}+\epsilon_{2}\|T x-y\|^{2}+\left(1-\epsilon_{1}-\epsilon_{2}\right)\|x-y\|^{2}
\end{gathered}
$$


for all $x, y \in C$. Replacing $x$ by $T^{k} y_{n}$ in equation (20), we have, for all $y \in C$ and $k=1,2, \ldots, n$,

$$
\begin{aligned}
\delta_{1} \| & T^{k+2} y_{n}-T y\left\|^{2}+\delta_{2}\right\| T^{k+1} y_{n}-T y\left\|^{2}+\left(1-\delta_{1}-\delta_{2}\right)\right\| T^{k} y_{n}-T y \|^{2} \\
\leq & \epsilon_{1}\left\|T^{k+2} y_{n}-y\right\|^{2}+\epsilon_{2}\left\|T^{k+1} y_{n}-y\right\|^{2}+\left(1-\epsilon_{1}-\epsilon_{2}\right)\left\|T^{k} y_{n}-y\right\|^{2} \\
\leq & \epsilon_{1}\left[\left\|T^{k+2} y_{n}-T y\right\|^{2}+\|T y-y\|^{2}+2\left\langle T^{k+2} y_{n}-T y, T y-y\right\rangle\right] \\
& +\epsilon_{2}\left[\left\|T^{k+1} y_{n}-T y\right\|^{2}+\|T y-y\|^{2}+2\left\langle T^{k+1} y_{n}-T y, T y-y\right\rangle\right] \\
& +\left(1-\epsilon_{1}-\epsilon_{2}\right)\left[\left\|T^{k} y_{n}-T y\right\|^{2}+\|T y-y\|^{2}+2\left\langle T^{k} y_{n}-T y, T y-y\right\rangle\right] .
\end{aligned}
$$

This implies that

$$
\begin{aligned}
0 \leq & \left(\epsilon_{1}-\delta_{1}\right)\left\|T^{k+2} y_{n}-T y\right\|^{2}+\|T y-y\|^{2}+2 \epsilon_{1}\left\langle T^{k+2} y_{n}-T y, T y-y\right\rangle \\
& +\left(\epsilon_{2}-\delta_{2}\right)\left\|T^{k+1} y_{n}-T y\right\|^{2}+2 \epsilon_{2}\left\langle T^{k+1} y_{n}-T y, T y-y\right\rangle \\
& +\left(\delta_{1}-\epsilon_{1}+\delta_{2}-\epsilon_{2}\right)\left\|T^{k} y_{n}-T y\right\|^{2} \\
& +2\left(1-\epsilon_{1}-\epsilon_{2}\right)\left\langle T^{k} y_{n}-T y, T y-y\right\rangle \\
\leq & \left(\epsilon_{1}-\delta_{1}\right)\left[\left\|T^{k+2} y_{n}-T y\right\|^{2}-\left\|T^{k} y_{n}-T y\right\|^{2}\right] \\
& +\left(\epsilon_{2}-\delta_{2}\right)\left[\left\|T^{k+1} y_{n}-T y\right\|^{2}-\left\|T^{k} y_{n}-T y\right\|^{2}\right]+\|T y-y\|^{2} \\
& +2\left\langle T^{k} y_{n}-T y+\epsilon_{1}\left(T^{k+2} y_{n}-T^{k} y_{n}\right)+\epsilon_{2}\left(T^{k+1} y_{n}-T^{k} y_{n}\right), T y-y\right\rangle .
\end{aligned}
$$

Summing up these inequalities (21) with respect to $k=0$ to $k=n-1$ and dividing by $n$, we have

$$
\begin{aligned}
0 \leq & \frac{\left(\epsilon_{1}-\delta_{1}\right)}{n}\left[\left\|T^{n+1} y_{n}-T y\right\|^{2}+\left\|T^{n} y_{n}-T y\right\|^{2}-\left\|T y_{n}-T y\right\|^{2}-\left\|y_{n}-T y\right\|^{2}\right] \\
& +\frac{\left(\epsilon_{2}-\delta_{2}\right)}{n}\left[\left\|T^{n} y_{n}-T y\right\|^{2}-\left\|y_{n}-T y\right\|^{2}\right] \\
& +\|T y-y\|^{2}+2\left\langle s_{n}-T y, T y-y\right\rangle \\
& +\frac{2}{n}\left\langle\epsilon_{1}\left(T^{n+1} y_{n}+T^{n} y_{n}-T y_{n}-y_{n}\right)+\epsilon_{2}\left(T^{n} y_{n}-y_{n}\right), T y-y\right\rangle .
\end{aligned}
$$

Replacing $n$ by $n_{k_{j}}$ and let $n_{k_{j}} \rightarrow \infty$. Then from equation (11), (16), and (22), we have $s_{n_{k_{j}}} \rightarrow w$, and

$$
0 \leq\|T y-y\|^{2}+2\langle w-T y, T y-y\rangle .
$$

Taking $y=w$ in the above inequality, we have

$$
0 \leq\|T w-w\|^{2}+2\langle w-T w, T w-w\rangle=\|T w-w\|^{2}-2\|T w-w\|^{2}=-\|T w-w\|^{2} .
$$

This implies that $w \in \operatorname{Fix}(T)$. Hence, $w \in \operatorname{Fix}(T) \cap\left(F_{1}+G_{1}\right)^{-1} 0 \cap\left(F_{2}+G_{2}\right)^{-1} 0$. Therefore, we have from equations (15) and (17)

$$
\limsup _{n \rightarrow \infty}\left\langle V \bar{x}_{0}-\theta, z_{n}-\bar{x}_{0}\right\rangle=\lim _{k \rightarrow \infty}\left\langle V \bar{x}_{0}-\theta, z_{n_{k}}-\bar{x}_{0}\right\rangle=\left\langle V \bar{x}_{0}-\theta, w-\bar{x}_{0}\right\rangle \geq 0 .
$$


By the same argument as the proof of Theorem 3.1 [16], we have

$$
\begin{aligned}
& \left\|x_{n+1}-\bar{x}_{0}\right\|^{2} \\
& \quad \leq\left[1-2\left(1-\alpha_{n}\right) \beta_{n} \tau\right]\left\|x_{n}-\bar{x}_{0}\right\|^{2}+2\left(1-\alpha_{n}\right) \beta_{n} \tau\left(\frac{\beta_{n} \tau\left\|x_{n}-\bar{x}_{0}\right\|^{2}}{2}\right. \\
& \left.\quad+\frac{\left\langle\theta_{n}-\theta, z_{n}-\bar{x}_{0}\right\rangle}{\tau}+\frac{\left\langle\theta-V \bar{x}_{0}, z_{n}-\bar{x}_{0}\right\rangle}{\tau}\right) .
\end{aligned}
$$

By equations (23), (24), assumptions, and Lemma 2.6, we know that $\lim _{n \rightarrow \infty} x_{n}=\bar{x}_{0}$, where

$$
\bar{x}_{0}=P_{\mathrm{Fix}(T) \cap\left(F_{1}+G_{1}\right)^{-1} 0 \cap\left(F_{2}+G_{2}\right)^{-1} 0}\left(\bar{x}_{0}-V \bar{x}_{0}+\theta\right) .
$$

Case 2: Suppose that there exists $\left\{n_{i}\right\}$ of $\{n\}$ such that $\left\|x_{n_{i}}-\bar{x}\right\| \leq\left\|x_{n_{i}+1}-\bar{x}\right\|$ for all $i \in \mathbb{N}$. By Lemma 2.5 , there exists a nondecreasing sequence $\left\{m_{j}\right\}$ in $\mathbb{N}$ such that $m_{j} \rightarrow \infty$ and

$$
\left\|x_{m_{j}}-\bar{x}\right\| \leq\left\|x_{m_{j}+1}-\bar{x}\right\| \quad \text { and } \quad\left\|x_{j}-\bar{x}\right\| \leq\left\|x_{m_{j}+1}-\bar{x}\right\| .
$$

Hence, it follows from equations (7) and (25) that

$$
\begin{aligned}
(1- & \left.\alpha_{m_{j}}\right) \alpha_{m_{j}}\left\|s_{m_{j}}-x_{m_{j}}\right\|^{2} \\
\leq & 2\left(1-\alpha_{m_{j}}\right) \beta_{m_{j}}\left\langle\theta_{m_{j}}, x_{m_{j}}-\bar{x}\right\rangle-2\left(1-\alpha_{m_{j}}\right) \beta_{m_{j}}\left\langle V s_{m_{j}}, x_{m_{j}}-\bar{x}\right\rangle \\
& +\left(1-\alpha_{m_{j}}\right)^{2}\left[\beta_{m_{j}}^{2}\left\|\theta_{m_{j}}-V s_{m_{j}}\right\|^{2}+2 \beta_{m_{j}}\left\|\theta_{m_{j}}-V s_{m_{j}}\right\|\left\|s_{m_{j}}-x_{m_{j}}\right\|\right]
\end{aligned}
$$

for each $j \in \mathbb{N}$. Hence, it follows from equation (26), (i), and (ii) that

$$
\lim _{j \rightarrow \infty}\left\|s_{m_{j}}-x_{m_{j}}\right\|=0
$$

We show that

$$
\limsup _{j \rightarrow \infty}\left\langle V \bar{x}_{0}-\theta, z_{m_{j}}-\bar{x}_{0}\right\rangle \geq 0
$$

Without loss of generality, there exists a subsequence $\left\{z_{m_{j_{k}}}\right\}$ of $\left\{z_{m_{j}}\right\}$ such that $z_{m_{j_{k}}} \rightarrow w$ for some $w \in H$ and

$$
\limsup _{j \rightarrow \infty}\left\langle V \bar{x}_{0}-\theta, z_{m_{j}}-\bar{x}_{0}\right\rangle=\lim _{k \rightarrow \infty}\left\langle V \bar{x}_{0}-\theta, z_{m_{j_{k}}}-\bar{x}_{0}\right\rangle
$$

By a similar argument as in the proof of Case 1 , we have $w \in \operatorname{Fix}(T) \cap\left(F_{1}+G_{1}\right)^{-1} 0 \cap\left(F_{2}+\right.$ $\left.G_{2}\right)^{-1} 0$. Therefore, we have from equations (28) and (15)

$$
\limsup _{j \rightarrow \infty}\left\langle V \bar{x}_{0}-\theta, z_{m_{j}}-\bar{x}_{0}\right\rangle=\lim _{k \rightarrow \infty}\left\langle V \bar{x}_{0}-\theta, z_{m_{j_{k}}}-\bar{x}_{0}\right\rangle=\left\langle V \bar{x}_{0}-\theta, w-\bar{x}_{0}\right\rangle \geq 0 .
$$


Following a similar argument as in the proof of Case 1, we have

$$
\begin{aligned}
& \left\|x_{m_{j}+1}-\bar{x}_{0}\right\|^{2} \\
& \quad \leq\left[1-2\left(1-\alpha_{m_{j}}\right) \beta_{m_{j}} \tau\right]\left\|x_{m_{j}}-\bar{x}_{0}\right\|^{2}+\left(1-\alpha_{m_{j}}\right)\left(\beta_{m_{j}} \tau\right)^{2}\left\|x_{m_{j}}-\bar{x}_{0}\right\|^{2} \\
& \quad+2 \beta_{m_{j}}\left(1-\alpha_{m_{j}}\right)\left\langle\theta_{n}-\theta, z_{m_{j}}-\bar{x}_{0}\right\rangle+2 \beta_{m_{j}}\left(1-\alpha_{m_{j}}\right)\left\langle\theta-V \bar{x}_{0}, z_{m_{j}}-\bar{x}_{0}\right\rangle .
\end{aligned}
$$

From $\left\|x_{m_{j}}-\bar{x}\right\| \leq\left\|x_{m_{j}+1}-\bar{x}\right\|$, we have

$$
\begin{aligned}
2(1 & \left.-\alpha_{m_{j}}\right) \beta_{m_{j}} \tau\left\|x_{m_{j}}-\bar{x}_{0}\right\|^{2} \\
\leq & \left(1-\alpha_{m_{j}}\right)\left(\beta_{m_{j}} \tau\right)^{2}\left\|x_{m_{j}}-\bar{x}_{0}\right\|^{2}+2 \beta_{m_{j}}\left(1-\alpha_{m_{j}}\right)\left\langle\theta_{n}-\theta, z_{m_{j}}-\bar{x}_{0}\right\rangle \\
& +2 \beta_{m_{j}}\left(1-\alpha_{m_{j}}\right)\left\langle\theta-V \bar{x}_{0}, z_{m_{j}}-\bar{x}_{0}\right\rangle .
\end{aligned}
$$

Since $\left(1-\alpha_{m_{j}}\right) \beta_{m_{j}}>0$, we have

$$
2 \tau\left\|x_{m_{j}}-\bar{x}_{0}\right\|^{2} \leq \beta_{m_{j}} \tau\left\|x_{m_{j}}-\bar{x}_{0}\right\|^{2}+2\left\langle\theta_{n}-\theta, z_{m_{j}}-\bar{x}_{0}\right\rangle+2\left\langle\theta-V \bar{x}_{0}, z_{m_{j}}-\bar{x}_{0}\right\rangle .
$$

By equations (29), (32), and the assumptions, we know that

$$
\lim _{j \rightarrow \infty}\left\|x_{m_{j}}-\bar{x}_{0}\right\|=0
$$

By (6), (27), and the assumptions, we know that

$$
\lim _{j \rightarrow \infty}\left\|x_{m_{j+1}}-x_{m_{j}}\right\|=0
$$

Thus, we have

$$
\lim _{j \rightarrow \infty}\left\|x_{m_{j+1}}-\bar{x}_{0}\right\|=0
$$

By equations (25) and (33),

$$
\lim _{j \rightarrow \infty}\left\|x_{j}-\bar{x}_{0}\right\| \leq \lim _{j \rightarrow \infty}\left\|x_{m_{j+1}}-\bar{x}_{0}\right\|=0 .
$$

Thus, the proof is completed.

\section{Remark 3.1}

(i) The assumptions, method, conclusion, and applications of Theorem 3.1 are different from Theorem 3.1 in [21] and [22]. In Theorem 3.1, Lemma 2.5 is used to prove the result, but in [21] and [22] we did not use this lemma.

(ii) The assumptions, method, and conclusion of Theorem 3.1 are different from Theorem 3.1 [16]. In Theorem 3.1 [16], $T$ is a quasi-nonexpansive with the demiclosed property, but in Theorem 3.1, $T$ is a 2-generalized hybrid mapping, and by Example 2.2, we know that $T$ does not satisfy the demiclosed property. Therefore Theorem 3.1 [16] cannot apply for a 2-generalized hybrid mapping. 


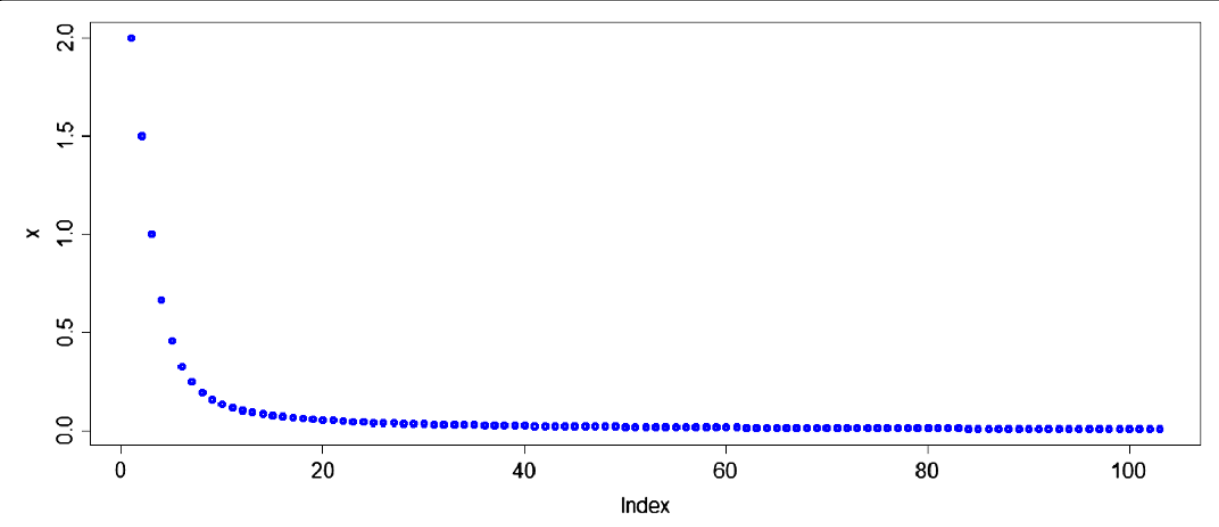

Figure $1 x$-axis: index; $y$-axis: $x_{n}$.

Example 3.1 Let $T$ be the same as Example 2.1. Let $\alpha_{n}=1 / 2, \beta_{n}=1 / n, \theta_{n}=1, V x=x$, $G_{1} x=F_{1} x=2 x, G_{2} x=F_{2} x=3 x, r_{n}=1 / 2, \lambda_{n}=1 / 2$. Then $V, G_{1}, G_{2}, F_{1}, F_{2}$ satisfy all conditions of Theorem 3.1 and $\operatorname{Fix}(T) \cap\left(F_{1}+G_{1}\right)^{-1} 0 \cap\left(F_{2}+G_{2}\right)^{-1} 0=\{0\}$, and if we let $x_{1}=2.000000000$, we see the following numerical results and graph (see Figure 1) demonstrating Theorem 3.1:

$\begin{array}{llllll}n=1-5 & 2.000000000 & 1.500000000 & 1.000000000 & 0.666666667 & 0.458333333 \\ n=6-10 & 0.329166667 & 0.247916667 & 0.195386905 & 0.160193452 & 0.135652282 \\ n=11-15 & 0.117826141 & 0.104367616 & 0.093850475 & 0.085386776 & 0.078407674\end{array}$

$\cdots$

Besides, we know the following.

If $\left|x_{n}-x_{n-1}\right|<10^{-3}$, then $n=35$; if $\left|x_{n}-x_{n-1}\right|<10^{-4}$, then $n=103$; if $\left|x_{n}-x_{n-1}\right|<10^{-5}$, then $n=319$; if $\left|x_{n}-x_{n-1}\right|<10^{-6}$, then $n=1,003$.

For $i=1,2$, let $F_{i}=0, G_{i}=\partial i_{C}$, and $\lambda_{n}=r_{n}=1$ for all $n \in \mathbb{N}$ in Theorem 3.1. Furthermore, put $\theta_{n}=\theta$, and $V(x)=x$ for all $x \in H_{1}$; we obtain the following theorem which generalizes Theorem 4.1 in [10].

Theorem 3.2 Let $T: C \rightarrow H_{1}$ be a 2-generalized hybrid mapping such that $F(T) \neq \emptyset$. Let $\theta \in C$, and $\left\{x_{n}\right\} \subset C$ be defined by

$$
\left\{\begin{array}{l}
x_{1} \in C \text { chosen arbitrarily, } \\
s_{n}=\frac{1}{n} \sum_{k=0}^{n-1} T^{k} x_{n} \\
x_{n+1}=\alpha_{n} x_{n}+\left(1-\alpha_{n}\right)\left(\beta_{n} \theta+\left(1-\beta_{n}\right) s_{n}\right)
\end{array}\right.
$$

for each $n \in \mathbb{N},\left\{\alpha_{n}\right\} \subset(0,1)$, and $\left\{\beta_{n}\right\} \subset(0,1)$. Assume that $0<\liminf _{n \rightarrow \infty} \alpha_{n} \leq$ $\lim \sup _{n \rightarrow \infty} \alpha_{n}<1$ and $\lim _{n \rightarrow \infty} \beta_{n}=0$, and $\sum_{n=1}^{\infty} \beta_{n}=\infty$. Then $\lim _{n \rightarrow \infty} x_{n}=\bar{x}$, where $\bar{x}=P_{\operatorname{Fix}(T)} \theta$. 


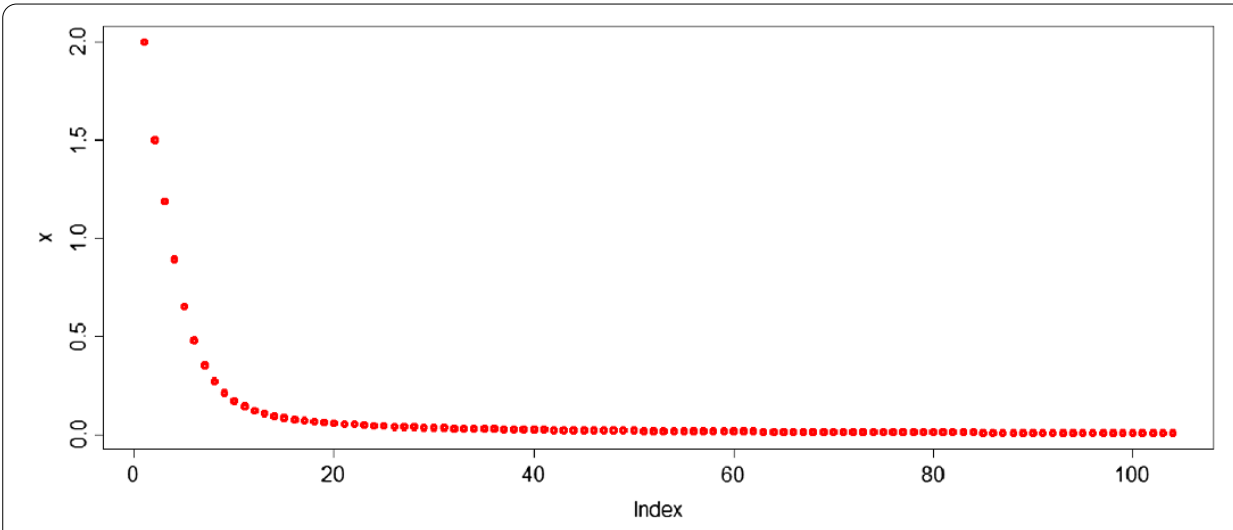

Figure $2 x$-axis: index; $y$-axis: $x_{n}$.

Example 3.2 Let $T$ be the same as Example 2.1. Let $\alpha_{n}=1 / 2, \beta_{n}=1 / n, \theta=1$. Then the following numerical results and graph (see Figure 2) demonstrate Theorem 3.2:

$\begin{array}{llllll}n=1-5 & 2.000000000 & 1.500000000 & 1.187500000 & 0.892361111 & 0.654839410 \\ n=6-10 & 0.479806858 & 0.356556683 & 0.271536914 & 0.213118132 & 0.172638974 \\ n=11-15 & 0.144088241 & 0.123452725 & 0.108108238 & 0.096353820 & 0.087086603 \\ n=16-20 & 0.079585996 & 0.073374619 & 0.068130205 & 0.063630247 & 0.059717263\end{array}$

Besides, we know the following.

If $\left|x_{n}-x_{n-1}\right|<10^{-3}$, then $n=36$; if $\left|x_{n}-x_{n-1}\right|<10^{-4}$, then $n=104$; if $\left|x_{n}-x_{n-1}\right|<10^{-5}$, then $n=320$; if $\left|x_{n}-x_{n-1}\right|<10^{-6}$, then $n=1,004$.

\section{Mathematical programming with multiple sets split feasibility constraints}

Let $H_{1}$ be a Hilbert space, let $f$ be a proper lower semicontinuous convex function of $H_{1}$ into $(-\infty, \infty)$. The subdifferential $\partial f$ of $f$ is defined as follows:

$$
\partial f(x)=\left\{z \in H_{1}: f(x)+\langle z, y-x\rangle \leq f(y), \forall y \in H_{1}\right\}
$$

for all $x \in H_{1}$. From Rockafellar [23], we know that $\partial f$ is a maximal monotone operator. Let $C$ be a nonempty closed convex subset of a real Hilbert space $H_{1}$, and $i_{C}$ be the indicator function of $C$, i.e.

$$
i_{C} x= \begin{cases}0 & \text { if } x \in C, \\ \infty & \text { if } x \notin C .\end{cases}
$$

Furthermore, we also define the normal cone $N_{C} u$ of $C$ at $u$ as follows:

$$
N_{C} u=\left\{z \in H_{1}:\langle z, v-u\rangle \leq 0, \forall v \in C\right\} .
$$

Then $i_{C}$ is a proper lower semicontinuous convex function on $H$, and the subdifferential $\partial i_{C}$ of $i_{C}$ is a maximal monotone operator. Thus, we can define the resolvent $J_{\lambda}^{\partial i_{C}}$ of $\partial i_{C}$ for $\lambda>0$, i.e.

$$
J_{\lambda}^{\partial i_{C}} x=\left(I+\lambda \partial i_{C}\right)^{-1} x
$$


for all $x \in H$. Since

$$
\begin{aligned}
\partial i_{C} x & =\left\{z \in H_{1}: i_{C} x+\langle z, y-x\rangle \leq i_{C} y, \forall y \in H_{1}\right\} \\
& =\left\{z \in H_{1}:\langle z, y-x\rangle \leq 0, \forall y \in C\right\} \\
& =N_{C} x
\end{aligned}
$$

for all $x \in C$, we have

$$
\begin{aligned}
u=J_{\lambda}^{\partial i_{C} x} & \Leftrightarrow x \in u+\lambda \partial i_{C} u \quad \Leftrightarrow \quad x-u \in \lambda N_{C} u \\
& \Leftrightarrow \quad\langle x-u, y-u\rangle \leq 0, \quad \forall y \in C \\
& \Leftrightarrow u=P_{C} x .
\end{aligned}
$$

The equilibrium problem is to find $z \in C$ such that

$$
g(z, y) \geq 0 \quad \text { for each } y \in C .
$$

The solutions set of the equilibrium problem (EP) is denoted by $E P(g)$. For solving the equilibrium problem, let us assume that the bifunction $g: C \times C \rightarrow \mathbb{R}$ satisfies the following conditions:

(A1) $g(x, x)=0$ for each $x \in C$;

(A2) $g$ is monotone, i.e., $g(x, y)+g(y, x) \leq 0$ for any $x, y \in C$;

(A3) for each $x, y, z \in C, \lim _{t \downarrow 0} g(t z+(1-t) x, y) \leq g(x, y)$;

(A4) for each $x \in C$, the scalar function $y \rightarrow g(x, y)$ is convex and lower semicontinuous.

Lemma 4.1 [24,25] Let $g: C \times C \rightarrow \mathbb{R}$ be a bifunction which satisfies conditions (A1)-(A4). Let $r>0$ and $x \in C$. Then there exists $z \in C$ such that

$$
g(z, y)+\frac{1}{r}\langle y-z, z-x\rangle \geq 0 \quad \text { for all } y \in C .
$$

Furthermore, if

$$
T_{r}^{g}(x):=\left\{z \in C: g(z, y)+\frac{1}{r}\langle y-z, z-x\rangle \geq 0 \text { for all } y \in C\right\},
$$

then we have:

(i) $T_{r}^{g}$ is single-valued;

(ii) $T_{r}^{g}$ is a firmly nonexpansive mapping;

(iii) $E P(g)$ is a closed convex subset of $C$;

(iv) $E P(g)=\operatorname{Fix}\left(T_{r}^{g}\right)$.

We call such $T_{r}^{g}$ the resolvent of $g$ for $r>0$. Throughout these section, we use these notations and assumptions unless specified otherwise.

Takahashi et al. [26] gave the following lemma. 
Lemma 4.2 [26] Let $g: C \times C \rightarrow \mathbb{R}$ be a bifunction satisfying the conditions (A1)-(A4). Define $A_{g}$ as follows:

$$
A_{g} x= \begin{cases}\left\{z \in H_{1}: g(x, y) \geq\langle y-x, z\rangle, \forall y \in C\right\} & \text { if } x \in C ; \\ \varnothing & \text { if } x \notin C .\end{cases}
$$

Then, $E P(g)=A_{g}^{-1} 0$ and $A_{g}$ is a maximal monotone operator with the domain of $A_{g} \subset C$. Furthermore, for any $x \in H_{1}$ and $r>0$, the resolvent $T_{r}^{g}$ of $g$ coincides with the resolvent of $A_{g}$, i.e., $T_{r}^{g} x=\left(I+r A_{g}\right)^{-1} x$.

Let $C, Q$, and $Q^{\prime}$ be nonempty closed convex subsets of real Hilbert spaces $H_{1}, H_{2}$, and $H_{3}$, respectively, let $G_{i}$ be a maximal monotone mapping on $H_{1}$ such that the domains of $G_{i}$ is included in $C$ for each $i=1,2$. Let $J_{\lambda}^{G_{1}}=\left(I+\lambda G_{1}\right)^{-1}$ and $J_{r}^{G_{2}}=\left(I+r G_{2}\right)^{-1}$ for each $\lambda>0$ and $r>0$, let $L_{1}$ be a $\kappa_{1}$-inverse-strongly monotone mapping of $C$ into $H_{1}$, let $L_{2}$ be a $\kappa_{2}$-inverse-strongly monotone mapping of $C$ into $H_{1}$, let $B$ be a $\nu$-inverse-strongly monotone mapping of $Q$ into $H_{2}$ and let $B^{\prime}$ be a $v^{\prime}$-inverse-strongly monotone mapping of $Q^{\prime}$ into $H_{3}$. Let $G$ be a maximal monotone mappings on $H_{2}$ such that the domain of $G$ is included in $Q$ and let $G^{\prime}$ be maximal a monotone mappings on $H_{3}$ such that the domain of $G^{\prime}$ is included in $Q^{\prime}$. Let $J_{\lambda^{\prime}}^{G}=\left(I+\lambda^{\prime} G\right)^{-1}$ and $J_{r^{\prime}}^{G^{\prime}}=\left(I+r^{\prime} G^{\prime}\right)^{-1}$ for each $\lambda^{\prime}>0$ and $r^{\prime}>0$. Let $A, A_{1}: H_{1} \rightarrow H_{2}$ be bounded linear operators, $A_{1}^{*}$ and $A^{*}$ the adjoints of $A_{1}$ and $A$ respectively, $A_{2}: H_{1} \rightarrow H_{3}$ a bounded linear operator, and $A_{2}^{*}$ the adjoint of $A_{2}$. Let $R_{i}$ be the spectral radius of the operator $A_{i}^{*} A_{i}$ for $i=1,2$, respectively, and $R$ the spectral radius of the operator $A^{*} A$. Let $I, I_{2}, I_{3}$ be the identity mappings of $H_{1}, H_{2}, H_{3}$, respectively. We use these notations throughout this section unless specified otherwise.

In order to study the convergence theorems for the solutions set of the multiple sets split monotone variational inclusion problem, we study the following essential problem (SFP-1):

Find $\bar{x} \in H_{1}$ such that $A \bar{x} \in(G+B)^{-1}(0)$.

Theorem 4.1 Given any $\bar{x} \in H_{1}$ we have the following.

(i) If $\bar{x}$ is a solution of (SFP-1), then $\left(I-\lambda A^{*}\left(I_{2}-U\right) A\right) \bar{x}=\bar{x}$, where $\lambda>0$, $U=J_{\sigma}^{G}\left(I_{2}-\sigma B\right)$ and $\sigma>0$.

(ii) Suppose that $U=J_{\sigma}^{G}\left(I_{2}-\sigma B\right), 0<\lambda<\frac{1}{R}, 0<\sigma<2 v$. Then $A^{*}\left(I_{2}-U\right) A$ is a $\frac{\kappa}{R}$-ism mapping, $J_{\sigma}^{G}\left(I_{2}-\sigma B\right)$, and $I-\lambda A^{*}\left(I_{2}-U\right) A$ are averaged for some $\kappa>\frac{1}{2}$. Suppose further that solution set of (SFP-1) is nonempty and $\left(I-\lambda A^{*}\left(I_{2}-U\right) A\right) \bar{x}=\bar{x}$. Then $\bar{x}$ is a solution of (SFP-1).

Proof (i) Suppose that $\bar{x} \in H_{1}$ is a solution of (SFP-1). Then $\bar{x} \in \mathcal{H}_{1}, A \bar{x} \in \operatorname{Fix}(U)$. It is easy to see that $\left(I-\lambda A^{*}\left(I_{2}-U\right) A\right) \bar{x}=\bar{x}$.

(ii) Since the solutions set of (SFP-1) is nonempty, there exists $\bar{w} \in H_{1}$ such that $A \bar{w} \in$ $F(U)$. Since $B$ is a $v$-inverse-strongly monotone mapping of $Q$ into $H_{2}$, it follows from Lemma 2.4(iii) and (iv) that

$$
J_{\sigma}^{G}\left(I_{2}-\sigma B\right) \text { is averaged. }
$$


By Lemma 2.4(iii), for some $\kappa>\frac{1}{2}$, we know that

$$
I_{2}-U=I_{2}-J_{\sigma}^{G}\left(I_{2}-\sigma B\right) \text { is } \kappa \text {-ism. }
$$

In Theorem 3.1 [9], Moudafi showed that

$$
A_{1}^{*}\left(I_{2}-U\right) A \text { is } \frac{\kappa}{R} \text {-ism. }
$$

By Lemma 2.4(iii) and $0<\lambda<\frac{1}{R}$, we know that

$$
I-\lambda A^{*}\left(I_{2}-U\right) A \text { is averaged }
$$

for some $\kappa>\frac{1}{2}$. Since

$$
\bar{x}=\left(I-\lambda A^{*}\left(I_{2}-U\right) A\right) \bar{x} .
$$

This implies

$$
A^{*}\left(I_{2}-U\right) A \bar{x}=0 .
$$

We know that $U(A \bar{x})=A \bar{x}+w_{1}$, with $A^{*} w_{1}=0$, which combined with the fact that $U(A \bar{w})=$ $A \bar{w}$ yields

$$
\|U(A \bar{x})-U(A \bar{w})\|^{2}=\left\|A \bar{x}+w_{1}-A \bar{w}\right\|^{2}=\|A \bar{x}-A \bar{w}\|^{2}+\left\|w_{1}\right\|^{2} .
$$

Since $U=J_{\sigma}^{G}\left(I_{2}-\sigma B\right)$ is a nonexpansive mapping and we have equation (41), we have $w_{1}=0$.

This implies that

$$
A \bar{x}=\operatorname{Fix}(U)=\operatorname{Fix}\left(J_{\sigma}^{G}\left(I_{2}-\sigma B\right)\right) .
$$

This shows that $\bar{x}$ is a solution of (SFP-1).

In the following theorem, we consider the multiple set split monotonic variational inclusion problem (MSSMVIP-1):

Find $\bar{x} \in H_{1}$ such that $\bar{x} \in G_{1}^{-1}(0) \cap G_{2}^{-1}(0), A_{1} \bar{x} \in(B+G)^{-1}(0)$, and $A_{2} \bar{x} \in\left(B^{\prime}+G^{\prime}\right)^{-1}(0)$.

That is,

Find $\bar{x} \in H_{1}$ such that $\bar{x} \in \operatorname{Fix}\left(J_{\lambda}^{G_{1}}\right) \cap \operatorname{Fix}\left(J_{r}^{G_{2}}\right), A_{1} \bar{x} \in \operatorname{Fix}\left(U_{1}\right)$ and $A_{2} \bar{x} \in \operatorname{Fix}\left(U_{2}\right)$ where $U_{1}=J_{\sigma}^{G}\left(I_{2}-\sigma B\right), U_{2}=J_{\sigma^{\prime}}^{G^{\prime}}\left(I_{3}-\sigma^{\prime} B^{\prime}\right)$.

Let $\Omega_{1}$ be the solutions set of (MSSMVIP-1). 
Theorem 4.2 Let $T: C \rightarrow H_{1}$ be a 2-generalized hybrid mapping. Suppose that $\Omega_{1}$ is the solutions set of (MSSMVIP-1) with $\operatorname{Fix}(T) \cap \Omega_{1} \neq \emptyset$. Take $\mu \in \mathbb{R}$ as follows:

$$
0<\mu<\frac{2 \bar{\gamma}}{L^{2}}
$$

Let $\left\{x_{n}\right\} \subset H_{1}$ be defined by

$$
\left\{\begin{array}{l}
x_{1} \in C \text { chosen arbitrarily, } \\
y_{n}=J_{\lambda}^{G_{1}}\left(I-\lambda A_{1}^{*}\left(I_{2}-U_{1}\right) A_{1}\right) J_{r}^{G_{2}}\left(I-r A_{2}^{*}\left(I_{3}-U_{2}\right) A_{2}\right) x_{n}, \\
s_{n}=\frac{1}{n} \sum_{k=0}^{n-1} T^{k} y_{n}, \\
x_{n+1}=\alpha_{n} x_{n}+\left(1-\alpha_{n}\right)\left(\beta_{n} \theta_{n}+\left(1-\beta_{n} V\right) s_{n}\right),
\end{array}\right.
$$

where $U_{1}=J_{\sigma}^{G}\left(I_{2}-\sigma B\right), U_{2}=J_{\sigma^{\prime}}^{G^{\prime}}\left(I_{3}-\sigma^{\prime} B^{\prime}\right),\left\{\alpha_{n}\right\} \subset(0,1),\left\{\beta_{n}\right\} \subset(0,1), r \in(0, \infty)$ and $\lambda \in$ $(0, \infty)$. We have

(i) $0<\liminf _{n \rightarrow \infty} \alpha_{n} \leq \limsup _{n \rightarrow \infty} \alpha_{n}<1$;

(ii) $\lim _{n \rightarrow \infty} \beta_{n}=0$, and $\sum_{n=1}^{\infty} \beta_{n}=\infty$;

(iii) $0<\lambda<\frac{1}{R_{1}}, 0<r<\frac{1}{R_{2}}, 0<\sigma<2 v$ and $0<\sigma^{\prime}<2 v^{\prime}$;

(iv) $\lim _{n \rightarrow \infty} \theta_{n}=\theta$ for some $\theta \in H_{1}$.

Then $\lim _{n \rightarrow \infty} x_{n}=\bar{x}$, where $\bar{x}=P_{\mathrm{Fix}(T) \cap \Omega_{1}}(\bar{x}-V \bar{x}+\theta)$. This point $\bar{x}$ is also a unique solution of the hierarchical variational inequality:

$$
\langle V \bar{x}-\theta, q-\bar{x}\rangle \geq 0, \quad \forall q \in \operatorname{Fix}(T) \cap \Omega_{1} .
$$

Proof Let $F_{1}=A_{1}^{*}\left(I_{2}-U_{1}\right) A_{1}, F_{2}=A_{2}^{*}\left(I_{3}-U_{2}\right) A_{2}, \lambda_{n}=\lambda$ and $r_{n}=r$ for all $n \in \mathbb{N}$ in Theorem 3.1. It follow from Theorem 4.1(ii) that $F_{i}$ is $\frac{\mu_{i}}{R_{i}}$-ivm for some $\mu_{i}>\frac{1}{2}$ and each $i=1,2$. Then algorithm (3.1) in Theorem 3.1 follows immediately from algorithm (4.2) in Theorem 4.2 .

Since $\operatorname{Fix}(T) \cap \Omega_{1}$ is nonempty, there exists $\bar{w} \in C$ such that

$$
\begin{gathered}
\bar{w} \in \operatorname{Fix}(T) \cap \operatorname{Fix}\left(J_{\lambda}^{G_{1}}\right) \cap \operatorname{Fix}\left(I-\lambda A_{1}^{*}\left(I_{2}-U_{1}\right) A_{1}\right) \\
\cap \operatorname{Fix}\left(J_{r}^{G_{2}}\right) \cap \operatorname{Fix}\left(I-r A_{2}^{*}\left(I_{3}-U_{2}\right) A_{2}\right) .
\end{gathered}
$$

This implies that

$$
\begin{gathered}
\bar{w} \in \operatorname{Fix}(T) \cap \operatorname{Fix}\left(J_{\lambda}^{G_{1}}\left(I-\lambda A_{1}^{*}\left(I_{2}-U_{1}\right) A_{1}\right)\right) \\
\cap \operatorname{Fix}\left(J_{r}^{G_{2}}\left(I-r A_{2}^{*}\left(I_{3}-U_{2}\right) A_{2}\right)\right) .
\end{gathered}
$$

That is,

$$
\bar{w} \in \operatorname{Fix}(T) \cap \operatorname{Fix}\left(J_{\lambda}^{G_{1}}\left(I-\lambda F_{1}\right)\right) \cap \operatorname{Fix}\left(J_{r}^{G_{2}}\left(I-r F_{2}\right)\right) .
$$

Hence,

$$
\bar{w} \in \operatorname{Fix}(T) \cap\left(G_{1}+F_{1}\right)^{-1} 0 \cap\left(G_{2}+F_{2}\right)^{-1} 0 \neq \emptyset .
$$


It follows from Theorem 3.1 that $\lim _{n \rightarrow \infty} x_{n}=\bar{x}$, where

$$
\bar{x}=P_{\mathrm{Fix}(T) \cap\left(F_{1}+G_{1}\right)^{-1} 0 \cap\left(F_{2}+G_{2}\right)^{-1} 0}(\bar{x}-V \bar{x}+\theta) .
$$

This point $\bar{x}$ is also a unique solution of the hierarchical variational inequality:

$$
\langle V \bar{x}-\theta, q-\bar{x}\rangle \geq 0, \quad \forall q \in \operatorname{Fix}(T) \cap\left(F_{1}+G_{1}\right)^{-1} 0 \cap\left(F_{2}+G_{2}\right)^{-1} 0 .
$$

If

$$
w \in \operatorname{Fix}(T) \cap\left(G_{1}+F_{1}\right)^{-1} 0 \cap\left(G_{2}+F_{2}\right)^{-1} 0 .
$$

By equations (44), (45), and (46), we know that

$$
w=T w, \quad w=J_{\lambda}^{G_{1}}\left(I-\lambda A_{1}^{*}\left(I_{2}-U_{1}\right) A_{1}\right) w, \quad w=J_{r}^{G_{2}}\left(I-r A_{2}^{*}\left(I_{3}-U_{2}\right) A_{2}\right) w .
$$

By $\Omega_{1} \neq \emptyset$, equation (48), and Lemma 2.4(v), we have

$$
\begin{aligned}
& w=T w, \quad w=J_{\lambda}^{G_{1}} w, \quad w=\left(I-\lambda A_{1}^{*}\left(I_{2}-U_{1}\right) A_{1}\right) w, \quad w=J_{r}^{G_{2}} w, \\
& w=\left(I-r A_{2}^{*}\left(I_{3}-U_{2}\right) A_{2}\right) w .
\end{aligned}
$$

It follows from Theorem 4.1(ii) that $w$ is a solution of (MSSMVIP-1). Therefore, $w \in$ Fix $(T) \cap \Omega_{1}$ and

$$
\operatorname{Fix}(T) \cap\left(G_{1}+F_{1}\right)^{-1} 0 \cap\left(G_{2}+F_{2}\right)^{-1} 0 \subseteq \operatorname{Fix}(T) \cap \Omega_{1} .
$$

Conversely, if $w \in \operatorname{Fix}(T) \cap \Omega_{1}$, by equations (43), (44), (45), and (46), we know that $w \in$ $\operatorname{Fix}(T) \cap\left(G_{1}+F_{1}\right)^{-1} 0 \cap\left(G_{2}+F_{2}\right)^{-1} 0$ and

$$
\operatorname{Fix}(T) \cap \Omega_{1} \subseteq \operatorname{Fix}(T) \cap\left(G_{1}+F_{1}\right)^{-1} 0 \cap\left(G_{2}+F_{2}\right)^{-1} 0 .
$$

Therefore, $\operatorname{Fix}(T) \cap \Omega_{1}=\operatorname{Fix}(T) \cap\left(G_{1}+F_{1}\right)^{-1} 0 \cap\left(G_{2}+F_{2}\right)^{-1} 0$ and the proof is completed.

Remark 4.1 Moudafi [9] studied a weak convergence theorem for the split monotone variational inclusion problem, while Theorem 4.2 is a strong convergence theorem for the multiply sets split monotone variational inclusion problem.

By Theorem 4.2, we study the mathematical programming problem with (MSSMVIP-1) and fixed point set constraints.

Theorem 4.3 In Theorem 4.2, let $h: C \rightarrow \mathbb{R}$ be a convex Gâteaux differential function with Gâteaux derivative $V$, and the assumption (iv) is replaced by $\lim _{n \rightarrow \infty} \theta_{n}=0$. Then $\lim _{n \rightarrow \infty} x_{n}=\bar{x}$, where $\bar{x}=P_{\mathrm{Fix}(T) \cap \Omega_{1}}(\bar{x}-V \bar{x})$. This point $\bar{x}$ is also a unique solution of the mathematical programming problem with (MSSMVIP-1) and fixed point constraints: $\min _{x \in \operatorname{Fix}(T) \cap \Omega_{1}} h(x)$. 
Proof Let $\theta=0$ in Theorem 4.2, by Theorem 4.2, we see that

$$
\langle V \bar{x}, q-\bar{x}\rangle \geq 0, \quad \forall q \in F(T) \cap \Omega_{1} .
$$

Since $h: C \rightarrow \mathbb{R}$ is a convex Gâteaux differential function with Gâteaux dirivitive $V$, we obtain

$$
\begin{aligned}
\langle V \bar{x}, y-\bar{x}\rangle & =\lim _{t \rightarrow 0} \frac{h(\bar{x}+t(y-\bar{x}))-h(\bar{x})}{t} \\
& =\lim _{t \rightarrow 0} \frac{h((1-t) \bar{x}+t y)-h(\bar{x})}{t} \\
& \leq \lim _{t \rightarrow 0} \frac{(1-t) h(\bar{x})+t h(y)-h(\bar{x})}{t} \\
& =h(y)-h(\bar{x})
\end{aligned}
$$

for all $y \in C$. By equations (50) and (51), it is easy to see that $h(\bar{x}) \leq h(q)$ for all $q \in \operatorname{Fix}(T) \cap$ $\Omega_{1}$.

If we put $h(x)=\frac{1}{2}\|x\|^{2}$ in Theorem 4.3, then $V=I$, and we have the following minimum norm of common solutions for (MSSMVIP-1) and Fix $(T)$.

Theorem 4.4 In Theorem 4.3, let the iteration process $\left\{x_{n+1}\right\}$ be replaced by

$$
x_{n+1}=\alpha_{n} x_{n}+\left(1-\alpha_{n}\right)\left(\beta_{n} \theta_{n}+\left(1-\beta_{n}\right) s_{n}\right), \quad n \in \mathbb{N} .
$$

Then $\lim _{n \rightarrow \infty} x_{n}=\bar{x}$, where $\bar{x}=P_{\mathrm{Fix}(T) \cap \Omega_{1}}(0)$. This point $\bar{x}$ is also a unique minimum solution of $\operatorname{Fix}(T) \cap \Omega_{1}: \min _{x \in \operatorname{Fix}(T) \cap \Omega_{1}}\|x\|$.

The multiple sets split variational inequality problem (MSSMVIP-2) is defined as follows:

Find $\bar{x} \in H_{1}$ such that $\bar{x} \in G_{1}^{-1}(0) \cap G_{2}^{-1}(0)$,

and

$$
\begin{aligned}
& \left\langle B\left(A_{1} \bar{x}\right), y-A_{1} \bar{x}\right\rangle \geq 0 \quad \text { for all } y \in Q, \\
& \left\langle B^{\prime}\left(A_{2} \bar{x}\right), y-A_{2} \bar{x}\right\rangle \geq 0 \quad \text { for all } y \in Q^{\prime} .
\end{aligned}
$$

That is,

$$
\text { Find } \bar{x} \in H_{1} \text { such that } \bar{x} \in \operatorname{Fix}\left(J_{\lambda}^{G_{1}}\right) \cap \operatorname{Fix}\left(J_{r}^{G_{2}}\right)
$$

and

$$
A_{1} \bar{x} \in \operatorname{Fix}\left(P_{Q}(I-\sigma B)\right) \text { and } A_{2} \bar{x} \in \operatorname{Fix}\left(P_{Q^{\prime}}\left(I-\sigma^{\prime} B^{\prime}\right)\right) .
$$

By Theorem 4.2, we can study a variational inequality problem with the split variational inequality (MSSMVIP-2) and fixed point set constraints. 
Theorem 4.5 In Theorem 4.2, let $U_{1}=J_{\sigma}^{G}(I-\sigma B), U_{2}=J_{\sigma^{\prime}}^{G^{\prime}}\left(I-\sigma^{\prime} B^{\prime}\right)$ be replaced by $U_{1}=P_{Q}(I-\sigma B), U_{2}=P_{Q^{\prime}}\left(I-\sigma^{\prime} B^{\prime}\right)$, respectively. Suppose that the set of solutions for (MSSMVIP-2) is $\Omega_{2}$ and $\operatorname{Fix}(T) \cap \Omega_{2} \neq \emptyset$. Then $\lim _{n \rightarrow \infty} x_{n}=\bar{x}$, where $\bar{x}=P_{\operatorname{Fix}(T) \cap \Omega_{2}}(\bar{x}-$ $V \bar{x}+\theta$ ). This point $\bar{x}$ is also a unique solution of the hierarchical variational inequality:

$$
\langle V \bar{x}-\theta, q-\bar{x}\rangle \geq 0, \quad \forall q \in \operatorname{Fix}(T) \cap \Omega_{2} .
$$

Proof Let $G=\partial i_{Q}$ and $G^{\prime}=\partial i_{Q^{\prime}}$ in Theorem 4.2, then, by equation (34), we have $J_{\sigma}^{G}(I-$ $\sigma B)=P_{Q}(I-\sigma B), J_{\sigma^{\prime}}^{G^{\prime}}\left(I-\sigma^{\prime} B^{\prime}\right)=P_{Q^{\prime}}\left(I-\sigma^{\prime} B^{\prime}\right)$. Since $\operatorname{Fix}(T) \cap \Omega_{2} \neq \emptyset$, there exists $\bar{w} \in C$ such that we can find

$$
\bar{w} \in H_{1} \text { such that } \bar{w} \in \operatorname{Fix}\left(J_{\lambda}^{G_{1}}\right) \cap \operatorname{Fix}\left(J_{r}^{G_{2}}\right)
$$

and

$$
A_{1} \bar{w} \in \operatorname{Fix}\left(P_{Q}(I-\sigma B)\right) \quad \text { and } \quad A_{2} \bar{w} \in \operatorname{Fix}\left(P_{Q^{\prime}}\left(I-\sigma^{\prime} B^{\prime}\right)\right) .
$$

This implies that

$$
A_{1} \bar{w} \in \operatorname{Fix}\left(J_{\sigma}^{G}(I-\sigma B)\right) \text { and } A_{2} \bar{w} \in \operatorname{Fix}\left(J_{\sigma^{\prime}}^{G^{\prime}}\left(I-\sigma^{\prime} B^{\prime}\right)\right) \text {. }
$$

Therefore, $\bar{w} \in \operatorname{Fix}(T) \cap \Omega_{1} \neq \emptyset$. It follows from Theorem 4.2 that $\lim _{n \rightarrow \infty} x_{n}=\bar{x}$, where $\bar{x}=$ $P_{\mathrm{Fix}(T) \cap \Omega_{1}}(\bar{x}-V \bar{x}+\theta)$. This point $\bar{x}$ is also a unique solution of the hierarchical variational inequality:

$$
\langle V \bar{x}-\theta, q-\bar{x}\rangle \geq 0, \quad \forall q \in \operatorname{Fix}(T) \cap \Omega_{1} .
$$

By equations (57), (58), and (59), $\lim _{n \rightarrow \infty} x_{n}=\bar{x}$, where $\bar{x}=P_{\mathrm{Fix}(T) \cap \Omega_{2}}(\bar{x}-V \bar{x}+\theta)$. This point $\bar{x}$ is also a unique solution of the hierarchical variational inequality:

$$
\langle V \bar{x}-\theta, q-\bar{x}\rangle \geq 0, \quad \forall q \in \operatorname{Fix}(T) \cap \Omega_{2} .
$$

Remark 4.2 Censor et al. [27] studied a weak convergence theorem for the split variational inequalities problem with the additional assumption, while Theorem 4.5 studies a strong convergence theorem for multiply sets split variational inequalities problem without this additional assumption.

By Theorem 4.5, we study a mathematical programming problem with (MSSMVIP-2) and fixed point set constraints.

Theorem 4.6 In Theorem 4.5, let $h: C \rightarrow \mathbb{R}$ be a convex Gâteaux differential function with Gâteaux derivative $V$, and the assumption (iv) is replaced by $\lim _{n \rightarrow \infty} \theta_{n}=0$. Then $\lim _{n \rightarrow \infty} x_{n}=\bar{x}$, where $\bar{x}=P_{\mathrm{Fix}(T) \cap \Omega_{2}}(\bar{x}-V \bar{x})$. This point $\bar{x}$ is also a unique solution of the following mathematical programming problem with (MSSMVIP-2) and fixed point constraints:

$$
\min _{x \in \operatorname{Fix}(T) \cap \Omega_{2}} h(x) .
$$


Proof By Theorem 4.5 and following the same argument as in the proof of Theorem 4.3, we see that the proof is complete.

In the following theorem, we consider the following split monotonic variational inclusion problem (MSSMVIP-3):

Find $\bar{x} \in H_{1}$ such that $\bar{x} \in G_{2}^{-1}(0), \bar{x} \in\left(G_{1}+F_{1}\right)^{-1} 0$, and $A_{2} \bar{x} \in\left(B^{\prime}+G^{\prime}\right)^{-1}(0)$.

That is,

Find $\bar{x} \in H_{1}$ such that $\bar{x} \in \operatorname{Fix}\left(J_{\lambda}^{G_{1}}\left(I-\lambda F_{1}\right)\right) \cap \operatorname{Fix}\left(J_{r}^{G_{2}}\right)$, and $A_{2} \bar{x} \in \operatorname{Fix}\left(U_{2}\right)$

where $U_{2}=J_{\sigma^{\prime}}^{G^{\prime}}\left(I_{3}-\sigma^{\prime} B^{\prime}\right)$.

Let $\Omega_{3}$ be the solutions set of (MSSMVIP-3).

Theorem 4.7 Let $T: C \rightarrow H_{1}$ be a 2-generalized hybrid mapping. Suppose that $\Omega_{3}$ is the solutions set of (MSSMVIP-3) with $\operatorname{Fix}(T) \cap \Omega_{3} \neq \emptyset$. Take $\mu \in \mathbb{R}$ as follows:

$$
0<\mu<\frac{2 \bar{\gamma}}{L^{2}}
$$

Let $\left\{x_{n}\right\} \subset H_{1}$ be defined by

$$
\left\{\begin{array}{l}
x_{1} \in C \text { chosen arbitrarily, } \\
y_{n}=J_{\lambda}^{G_{1}}\left(I-\lambda F_{1}\right) J_{r}^{G_{2}}\left(I-r A_{2}^{*}\left(I_{3}-U_{2}\right) A_{2}\right) x_{n}, \\
s_{n}=\frac{1}{n} \sum_{k=0}^{n-1} T^{k} y_{n}, \\
x_{n+1}=\alpha_{n} x_{n}+\left(1-\alpha_{n}\right)\left(\beta_{n} \theta_{n}+\left(1-\beta_{n} V\right) s_{n}\right),
\end{array}\right.
$$

where $U_{2}=J_{\sigma^{\prime}}^{G^{\prime}}\left(I_{3}-\sigma^{\prime} B^{\prime}\right),\left\{\alpha_{n}\right\} \subset(0,1),\left\{\beta_{n}\right\} \subset(0,1), r \in(0, \infty)$ and $\lambda \in(0, \infty)$.

(i) $0<\liminf _{n \rightarrow \infty} \alpha_{n} \leq \limsup _{n \rightarrow \infty} \alpha_{n}<1$;

(ii) $\lim _{n \rightarrow \infty} \beta_{n}=0$, and $\sum_{n=1}^{\infty} \beta_{n}=\infty$;

(iii) $0<\lambda<2 \kappa_{1}, 0<r<\frac{1}{R_{2}}$ and $0<\sigma^{\prime}<2 v^{\prime}$;

(iv) $\lim _{n \rightarrow \infty} \theta_{n}=\theta$ for some $\theta \in H_{1}$.

Then $\lim _{n \rightarrow \infty} x_{n}=\bar{x}$, where $\bar{x}=P_{\mathrm{Fix}(T) \cap \Omega_{3}}(\bar{x}-V \bar{x}+\theta)$. This point $\bar{x}$ is also a unique solution of the hierarchical variational inequality:

$$
\langle V \bar{x}-\theta, q-\bar{x}\rangle \geq 0, \quad \forall q \in \operatorname{Fix}(T) \cap \Omega_{3} .
$$

Proof Let $F_{2}=A_{2}^{*}\left(I-U_{2}\right) A_{2}, \lambda_{n}=\lambda$ and $r_{n}=r$ for all $n \in \mathbb{N}$ in Theorem 3.1. It follows from Theorem 4.1(ii) that $F_{2}$ is $\frac{\mu_{i}}{R_{i}}$-ivm for some $\mu_{i}>\frac{1}{2}$. Then algorithm (3.1) in Theorem 3.1 follows immediately from algorithm (4.7) in Theorem 4.7.

Since $\operatorname{Fix}(T) \cap \Omega_{3}$ is nonempty, there exists $\bar{w} \in C$ such that

$$
\bar{w} \in \operatorname{Fix}(T) \cap \operatorname{Fix}\left(J_{\lambda}^{G_{1}}\left(I-\lambda F_{1}\right)\right) \cap \operatorname{Fix}\left(J_{r}^{G_{2}}\right) \cap \operatorname{Fix}\left(I-r A_{2}^{*}\left(I-U_{2}\right) A_{2}\right) .
$$

This implies that

$$
\bar{w} \in \operatorname{Fix}(T) \cap \operatorname{Fix}\left(J_{\lambda}^{G_{1}}\left(I-\lambda F_{1}\right)\right) \cap \operatorname{Fix}\left(J_{r}^{G_{2}}\left(I-r A_{2}^{*}\left(I-U_{2}\right) A_{2}\right)\right) .
$$


That is,

$$
\bar{w} \in \operatorname{Fix}(T) \cap \operatorname{Fix}\left(J_{\lambda}^{G_{1}}\left(I-\lambda F_{1}\right)\right) \cap \operatorname{Fix}\left(J_{r}^{G_{2}}\left(I-r F_{2}\right)\right) .
$$

Hence,

$$
\bar{w} \in \operatorname{Fix}(T) \cap\left(G_{1}+F_{1}\right)^{-1} 0 \cap\left(G_{2}+F_{2}\right)^{-1} 0 \neq \emptyset .
$$

It follows from Theorem 3.1 that $\lim _{n \rightarrow \infty} x_{n}=\bar{x}$, where

$$
\bar{x}=P_{\mathrm{Fix}(T) \cap\left(F_{1}+G_{1}\right)^{-1} 0 \cap\left(F_{2}+G_{2}\right)^{-1} 0}(\bar{x}-V \bar{x}+\theta) .
$$

This point $\bar{x}$ is also a unique solution of the hierarchical variational inequality:

$$
\langle V \bar{x}-\theta, q-\bar{x}\rangle \geq 0, \quad \forall q \in \operatorname{Fix}(T) \cap\left(F_{1}+G_{1}\right)^{-1} 0 \cap\left(F_{2}+G_{2}\right)^{-1} 0 .
$$

If

$$
w \in \operatorname{Fix}(T) \cap\left(G_{1}+F_{1}\right)^{-1} 0 \cap\left(G_{2}+F_{2}\right)^{-1} 0 .
$$

That is,

$$
w=T w, \quad w=J_{\lambda}^{G_{1}}\left(I-F_{1}\right) w \quad \text { and } \quad w=J_{r}^{G_{2}}\left(I-r A_{2}^{*}\left(I-U_{2}\right) A_{2}\right) w .
$$

By $\Omega_{3} \neq \emptyset$, equation (65) and Lemma 2.4(v), we have

$$
w=T w, \quad w=J_{\lambda}^{G_{1}}\left(I-\lambda F_{1}\right) w, \quad w=J_{r}^{G_{2}} w, \quad w=\left(I-r A_{2}^{*}\left(I_{3}-U_{2}\right) A_{2}\right) w .
$$

By $\Omega_{3} \neq \emptyset$, equation (66), and Theorem 4.1(ii), we see that $w$ is a solution of (MSSMVIP-3). Therefore, $w \in \operatorname{Fix}(T) \cap \Omega_{3}$ and $\operatorname{Fix}(T) \cap\left(G_{1}+F_{1}\right)^{-1} 0 \cap\left(G_{2}+F_{2}\right)^{-1} 0 \subseteq \operatorname{Fix}(T) \cap \Omega_{3}$. Conversely, if $w \in \operatorname{Fix}(T) \cap \Omega_{3}$, by equations (60), (61), (62), and (63), we know that $w \in \operatorname{Fix}(T) \cap\left(G_{1}+F_{1}\right)^{-1} 0 \cap\left(G_{2}+F_{2}\right)^{-1} 0$ and

$$
\operatorname{Fix}(T) \cap \Omega_{3} \subseteq \operatorname{Fix}(T) \cap\left(G_{1}+F_{1}\right)^{-1} 0 \cap\left(G_{2}+F_{2}\right)^{-1} 0
$$

Therefore, $\operatorname{Fix}(T) \cap \Omega_{3}=\operatorname{Fix}(T) \cap\left(G_{1}+F_{1}\right)^{-1} 0 \cap\left(G_{2}+F_{2}\right)^{-1} 0$ and the proof is completed.

Remark 4.3 Theorem 4.7 also improve Theorem 3.1 [9].

For each $i=1,2$, let $f_{i}: C \times C \rightarrow \mathbb{R}$ be a bifunction satisfying the conditions (A1)-(A4). The system of mixed type equilibria problem (MSSMVIP-4) is defined as follows.

Find $\bar{x} \in C$ such that $f_{1}(\bar{x}, x)+\left\langle x-\bar{x}, F_{1} \bar{x}\right\rangle \geq 0$ and $f_{2}(\bar{x}, x)+\left\langle x-\bar{x}, F_{2} \bar{x}\right\rangle \geq 0$ for all $x \in C$.

By Theorem 3.1 and Lemma 4.2, we study a variational inequality problem with (MSSMVIP-4) and fixed point set constraints. 
Theorem 4.8 Let $T: C \rightarrow H_{1}$ be a 2-generalized hybrid mapping. For each $i=1,2$, let $f_{i}: C \times C \rightarrow \mathbb{R}$ be a bifunction satisfying the conditions (A1)-(A4), and $J_{\lambda}^{A_{f_{1}}}, f_{r}^{A_{f_{2}}}$, defined as Lemma 4.2. Suppose that $\Omega_{4}$ is the solutions set of (MSSMVIP-4) with $\left(A_{f_{1}}+F_{1}\right)^{-1} 0 \cap$ $\left(A_{f_{2}}+F_{2}\right)^{-1} 0 \cap \operatorname{Fix}(T) \neq \emptyset$. Let $\left\{x_{n}\right\} \subset H$ be defined by

$$
\left\{\begin{array}{l}
x_{1} \in C \text { chosen arbitrarily } \\
y_{n}=J_{\lambda}^{A_{f_{1}}}\left(I-\lambda F_{1}\right) J_{r}^{A_{f_{2}}}\left(I-r F_{2}\right) x_{n} \\
s_{n}=\frac{1}{n} \sum_{k=0}^{n-1} T^{k} y_{n} \\
x_{n+1}=\alpha_{n} x_{n}+\left(1-\alpha_{n}\right)\left(\beta_{n} \theta_{n}+\left(1-\beta_{n} V\right) s_{n}\right)
\end{array}\right.
$$

where $\left\{\alpha_{n}\right\} \subset(0,1),\left\{\beta_{n}\right\} \subset(0,1), r \in(0, \infty)$ and $\lambda \in(0, \infty)$. Assume that:

(i) $0<\liminf _{n \rightarrow \infty} \alpha_{n} \leq \limsup _{n \rightarrow \infty} \alpha_{n}<1$;

(ii) $\lim _{n \rightarrow \infty} \beta_{n}=0$, and $\sum_{n=1}^{\infty} \beta_{n}=\infty$;

(iii) $0<a_{1} \leq \lambda \leq b_{1}<2 \kappa_{1}, 0<a_{2} \leq r \leq b_{2}<\kappa_{2}$;

(iv) $\lim _{n \rightarrow \infty} \theta_{n}=\theta$ for some $\theta \in H_{1}$.

Then $\lim _{n \rightarrow \infty} x_{n}=\bar{x}$, where $\bar{x}=P_{\mathrm{Fix}(T) \cap \Omega_{4}}(\bar{x}-V \bar{x}+\theta)$. This point $\bar{x}$ is also a unique solution of the hierarchical variational inequality:

$$
\langle V \bar{x}-\theta, q-\bar{x}\rangle \geq 0, \quad \forall q \in \operatorname{Fix}(T) \cap \Omega_{4} .
$$

Proof For each $i=1,2$, let $A_{f_{i}}$ be as in Lemma 4.2. By Lemma 4.2, we see that $A_{f_{i}}$ is a maximal monotone operator with the domain of $A_{f_{i}} \subset C$. Furthermore, for any $x \in H_{1}$ and $r>0$, the resolvent $T_{\lambda}^{f_{i}}$ of $f_{i}$ coincides with the resolvent of $A_{f_{i}}$, i.e.,

$$
T_{\lambda}^{f_{i}} x=\left(I+\lambda A_{f_{i}}\right)^{-1} x
$$

For $i=1,2$, let $G_{i}=A_{f_{i}}$ in Theorem 3.1. By equation (67), we have

$$
T_{\lambda}^{f_{i}} x=\left(I+\lambda A_{f_{1}}\right)^{-1} x=J_{\lambda}^{G_{1}} x, \quad T_{r}^{f_{2}} x=\left(I+r A_{f_{2}}\right)^{-1} x=J_{r}^{G_{2}} x .
$$

Then algorithm (3.1) in Theorem 3.1 follows immediately from algorithm (4.8) in Theorem 4.8 .

By equation (68), we have $\operatorname{Fix}\left(T_{\lambda}^{f_{1}}\right)=A_{f_{1}}^{-1} 0=\operatorname{Fix}\left(J_{\lambda}^{A_{f_{1}}}\right)$ and $\operatorname{Fix}\left(T_{r}^{f_{2}}\right)=A_{f_{2}}^{-1} 0=\operatorname{Fix}\left(J_{r}^{A_{f_{2}}}\right)$. It follows from Theorem 3.1 that $\lim _{n \rightarrow \infty} x_{n}=\bar{x}$, where

$$
\bar{x}=P_{\operatorname{Fix}(T) \cap \Omega_{1}}(\bar{x}-V \bar{x}+\theta) .
$$

This point $\bar{x}$ is also a unique solution of the hierarchical variational inequality:

$$
\langle V \bar{x}-\theta, q-\bar{x}\rangle \geq 0, \quad \forall q \in \operatorname{Fix}(T) \cap \Omega_{1} .
$$

Here $w \in\left(G_{1}+F_{1}\right)^{-1} 0 \cap\left(G_{2}+F_{2}\right)^{-1} 0$. That is, $w \in\left(A_{f_{1}}+F_{1}\right)^{-1} 0 \cap\left(A_{f_{2}}+F_{2}\right)^{-1} 0$. That is, $w \in \operatorname{Fix}\left(J_{\lambda}^{A_{f_{1}}}\left(I-\lambda F_{1}\right)\right) \cap \operatorname{Fix}\left(J_{r}^{A_{f_{2}}}\left(I-r F_{2}\right)\right)$. That is,

$$
f_{1}(w, x)+\left\langle x-w, F_{1} w\right\rangle \geq 0
$$


and

$$
f_{2}(w, x)+\left\langle x-w, F_{2} w\right\rangle \geq 0
$$

for all $x \in C$. Therefore, $\Omega_{1}=\Omega_{4}$ and the proof is complete.

By Theorem 4.2, we study a mathematical programming problem with (MSSMVIP-4) and fixed point set constraints.

Theorem 4.9 In Theorem 4.8, let $h: C \rightarrow \mathbb{R}$ be a convex Gâteaux differential function with Gâteaux derivative $V$, and let the assumption (iv) be replaced by $\lim _{n \rightarrow \infty} \theta_{n}=0$. Then $\lim _{n \rightarrow \infty} x_{n}=\bar{x}$, where $\bar{x}=P_{\mathrm{Fix}(T) \cap \Omega_{4}}(\bar{x}-V \bar{x})$. This point $\bar{x}$ is also a unique solution of the mathematical programming problem with (MSSMVIP-2) constraints:

$$
\min _{x \in \operatorname{Fix}(T) \cap \Omega_{4}} h(x) .
$$

For each $i=1,2$, let $f_{i}: C \times C \rightarrow \mathbb{R}$ and $g_{1}: Q \times Q \rightarrow \mathbb{R}, g_{2}: Q^{\prime} \times Q^{\prime} \rightarrow \mathbb{R}$ be bifunctions satisfying conditions (A1)-(A4). The multiple sets split system of mixed type equilibrium problems (MSSMVIP-5) is defined as follows.

Find $\bar{x} \in C$ such that $\bar{x} \in E P\left(f_{1}\right) \cap E P\left(f_{2}\right), g_{1}\left(A_{1} \bar{x}, y\right)+\left\langle y-A_{1} \bar{x}, B A_{1} \bar{x}\right\rangle \geq 0$ and

$$
g_{2}\left(A_{2} \bar{x}, y^{\prime}\right)+\left\langle y^{\prime}-A_{2} \bar{x}, B^{\prime} A_{2} \bar{x}\right\rangle \geq 0
$$

for all $y \in Q, y^{\prime} \in Q^{\prime}$.

By Theorem 4.2, we can study a variational inequality problem with (MSSMVIP-5) and fixed point set constraints.

Theorem 4.10 For each $i=1,2$, let $f_{i}: C \times C \rightarrow \mathbb{R}$ and $g_{1}: Q \times Q \rightarrow \mathbb{R}, g_{2}: Q^{\prime} \times Q^{\prime} \rightarrow \mathbb{R}$ be bifunctions satisfying the conditions (A1)-(A4). Let $T: C \rightarrow H_{1}$ be a 2-generalized hybrid mapping, and let $J_{\lambda}^{A_{f_{1}}}, J_{r}^{A_{f_{2}}}, J_{\sigma}^{A_{g_{1}}}, J_{\sigma}^{A_{g_{2}}}$ be defined as in Lemma 4.2. Suppose that $\Omega_{5}$ is the solutions set of (MSSMVIP-4) with $\Omega_{5} \cap \operatorname{Fix}(T) \neq \emptyset$. Let $\left\{x_{n}\right\} \subset H$ be defined by

$$
\left\{\begin{array}{l}
x_{1} \in C \text { chosen arbitrarily, } \\
y_{n}=J_{\lambda}^{A_{f_{1}}}\left(I-\lambda A_{1}^{*}\left(I_{2}-U_{1}\right) A_{1}\right) J_{\lambda}^{A_{f_{2}}}\left(I-r A_{2}^{*}\left(I_{3}-U_{2}\right) A_{2}\right) x_{n} \\
s_{n}=\frac{1}{n} \sum_{k=0}^{n-1} T^{k} y_{n} \\
x_{n+1}=\alpha_{n} x_{n}+\left(1-\alpha_{n}\right)\left(\beta_{n} \theta_{n}+\left(1-\beta_{n} V\right) s_{n}\right),
\end{array}\right.
$$

where $U_{1}=J_{\sigma}^{A g_{1}}\left(I_{2}-\sigma B\right), U_{2}=J_{\sigma^{\prime}}^{A g_{2}}\left(I_{3}-\sigma^{\prime} B^{\prime}\right)$. Then $\lim _{n \rightarrow \infty} x_{n}=\bar{x}$, where $\bar{x}=P_{\text {Fix }(T) \cap \Omega_{5}}(\bar{x}-$ $V \bar{x}+\theta)$. This point $\bar{x}$ is also a unique solution of the hierarchical variational inequality:

$$
\langle V \bar{x}-\theta, q-\bar{x}\rangle \geq 0, \quad \forall q \in \operatorname{Fix}(T) \cap \Omega_{5} .
$$

Proof For $i=1,2$, let $G_{i}=A_{f_{i}}, G=A_{g_{1}}$, and $G^{\prime}=A_{g_{2}}$ in Theorem 4.2. By Theorem 4.2 and following the same argument as in the proof of Theorem 4.8, we prove Theorem 4.10. 
For each $i=1,2$, let $f_{i}: C \times C \rightarrow \mathbb{R}$ and $g_{i}: Q \times Q \rightarrow \mathbb{R}$ be bifunctions satisfying conditions (A1)-(A4). The split mixed type equilibrium problem (MSSMVIP-6) is defined as follows.

Find $\bar{x} \in C$ such that $\bar{x} \in E P\left(f_{2}\right), f_{1}(\bar{x}, x)+\left\langle x-\bar{x}, F_{1} \bar{x}\right\rangle \geq 0$ and

$$
g_{2}\left(A_{2} \bar{x}, y\right)+\left\langle y-A_{2} \bar{x}, B^{\prime} A_{2} \bar{x}\right\rangle \geq 0
$$

for all $x \in C, y \in Q$.

Applying Theorem 4.7 and following a similar argument as in Theorem 4.10, we can study a variational inequality problem with (MSSMVIP-6) and with fixed point set constraints.

Theorem 4.11 For each $i=1,2$, let $f_{i}: C \times C \rightarrow \mathbb{R}$ and $g_{i}: Q \times Q \rightarrow \mathbb{R}$ be bifunctions satisfying conditions (A1)-(A4). Let $T: C \rightarrow H_{1}$ be a 2-generalized hybrid mapping, and $J_{\lambda}^{A_{f_{1}}}, J_{r}^{A_{f_{2}}}, J_{\sigma}^{A g_{1}}, J_{\sigma}^{A g_{2}}$ defined as in Lemma 4.2. Suppose that $\Omega_{6}$ is the solutions set of (MSSMVIP-6) with $\Omega_{6} \cap \operatorname{Fix}(T) \neq \emptyset$. Let $\left\{x_{n}\right\} \subset H$ be defined by

$$
\left\{\begin{array}{l}
x_{1} \in C \text { chosen arbitrarily, } \\
y_{n}=J_{\lambda}^{A_{f_{1}}}\left(I-\lambda F_{1}\right) J_{\lambda}^{A_{f_{2}}}\left(I-r A_{2}^{*}\left(I_{3}-U_{2}\right) A_{2}\right) x_{n}, \\
s_{n}=\frac{1}{n} \sum_{k=0}^{n-1} T^{k} y_{n}, \\
x_{n+1}=\alpha_{n} x_{n}+\left(1-\alpha_{n}\right)\left(\beta_{n} \theta_{n}+\left(1-\beta_{n} V\right) s_{n}\right),
\end{array}\right.
$$

where $U_{2}=J_{\sigma^{\prime}}^{A g_{2}}\left(I_{3}-\sigma^{\prime} B^{\prime}\right),\left\{\alpha_{n}\right\} \subset(0,1),\left\{\beta_{n}\right\} \subset(0,1), r \in(0, \infty)$ and $\lambda \in(0, \infty)$. Assume further that

(i) $0<\liminf _{n \rightarrow \infty} \alpha_{n} \leq \limsup _{n \rightarrow \infty} \alpha_{n}<1$;

(ii) $\lim _{n \rightarrow \infty} \beta_{n}=0$, and $\sum_{n=1}^{\infty} \beta_{n}=\infty$;

(iii) $0<\lambda<2 \kappa_{1}, 0<r<\frac{1}{R_{2}}$ and $0<\sigma^{\prime}<2 v^{\prime}$;

(iv) $\lim _{n \rightarrow \infty} \theta_{n}=\theta$ for some $\theta \in H_{1}$.

Then $\lim _{n \rightarrow \infty} x_{n}=\bar{x}$, where $\bar{x}=P_{\mathrm{Fix}(T) \cap \Omega_{6}}(\bar{x}-V \bar{x}+\theta)$. This point $\bar{x}$ is also a unique solution of the hierarchical variational inequality:

$$
\langle V \bar{x}-\theta, q-\bar{x}\rangle \geq 0, \quad \forall q \in \operatorname{Fix}(T) \cap \Omega_{6} .
$$

\section{Competing interests}

The authors declare no competing interests, except Prof. Z-TY was supported by Nan Kai University of Technology, and Prof. C-SC was supported by the National Science Council of Republic of China while he work on the publish.

Authors' contributions

Z-TY carry out the project, draft and revise the manuscript. L-JL design this research project, coordination, revise the paper. C-SC coordinate and the project and revise the paper, and give the numerical results. All authors read and approved the final manuscript.

\section{Author details}

'Department of Electronic Engineering, Nan Kai University of Technology, Nantou, 542, Taiwan. ${ }^{2}$ Department of Mathematics, National Changhua University of Education, Changhua, 50058, Taiwan. ${ }^{3}$ Department of Applied Mathematics, National Sun Yat-Sen University, Kaohsiung, Taiwan. 


\section{References}

1. Censor, Y, Elfving, T: A multiprojection algorithm using Bregman projection in a product space. Numer. Algorithms 8 , 221-239 (1994)

2. Byrne, C: Iterative oblique projection onto convex sets and the split feasibility problem. Inverse Probl. 18, 441-453 (2002)

3. Censor, Y, Bortfeld, T, Martin, B, Trofimov, A: A unified approach for inversion problems in intensity modulated radiation therapy. Phys. Med. Biol. 51, 2353-2365 (2003)

4. López, G, Martín-Márquez, V, Xu, HK: Iterative algorithms for the multiple-sets split feasibility problem. In: Censor, Y, Jiang, M, Wang, G (eds.) Biomedical Mathematics: Promising Directions in Imaging, Therapy Planning and Inverse Problems, pp. 243-279. Medical Physics Publishing, Madison (2010)

5. Stark, H: Image Recovery: Theory and Applications. Academic Press, New York (1987)

6. Censor, Y, Elfving, T, Kopf, N, Bortfeld, T: The multiple-sets split feasibility problem and its applications for inverse problems. Inverse Probl. 21, 2071-2084 (2005)

7. Xu, HK: Iterative methods for the split feasibility problem in infinite-dimensional Hilbert spaces. Inverse Probl. 26, $105018(2010)$

8. Lopez, G, Martín-Márquez, V, Wang, F, Xu, HK: Solving the split feasibility problem without prior knowledge of matrix. Inverse Probl. 28, 085004 (2012)

9. Moudafi, A: Split monotone variational inclusions. J. Optim. Theory Appl. 150, 275-283 (2011). doi:10.1007/s10957-011-9814-6

10. Maruyama, T, Takahashi, W, Yao, M: Fixed point and mean ergodic theorems for new nonlinear mappings in Hilbert spaces. J. Nonlinear Convex Anal. 12, 185-197 (2011)

11. Baillon, J-B: Un théorème de type ergodique pour les contractions non linéaires dans un espace de Hilbert. C. R. Acad. Sci. Paris Sér. A-B 280, 1511-1514 (1975)

12. Kocourek, $\mathrm{P}$, Takahashi, W, Yao, JC: Fixed point theorems and weak convergence theorems for generalized hybrid mappings in Hilbert spaces. Taiwan. J. Math. 14, 2497-2511 (2010)

13. Naraghirad, E, Lin, $\sqcup$ : Strong convergence theorems for generalized nonexpansive mappings on star shaped with applications to monotone variational inclusion problems. Preprint

14. Browder, FE: Fixed point theorems for noncompact mappings in Hilbert spaces. Proc. Natl. Acad. Sci. USA 53, 1272-1276 (1965)

15. Hojo, M, Takahashi, W, Termwuttipongc, I: Strong convergence theorems for 2-generalized hybrid mappings in Hilbert spaces. Nonlinear Anal. TMA 53(4), 2166-2176 (2012)

16. Yu, ZT, Lin, LJ: Hierarchical problems with applications to mathematical programming with multiple sets split feasibility constraints. Fixed Point Theory Appl. 2013, Article ID 283 (2013)

17. Takahashi, W: Nonlinear Functional Analysis-Fixed Point Theory and Its Applications. Yokohama Publishers, Yokohama (2000)

18. Combettes, PL: Solving monotone inclusions via compositions of nonexpansive averaged operators. Optimization 53, 475-504 (2004)

19. Maingé, PE: Strong convergence of projected subgradient methods for nonsmooth and nonstrictly convex minimization. Set-Valued Anal. 16, 899-912 (2008)

20. Aoyama, K, Kimura, Y, Takahashi, W, Toyoda, M: Approximation of common fixed points of a countable family of nonexpansive mappings in a Banach space. Nonlinear Anal. 67, 2350-2360 (2007)

21. Takahashi, W: Strong convergence theorems for maximal and inverse-strongly monotone mappings in Hilbert spaces and applications. J. Optim. Theory Appl., 157, 781-802 (2013)

22. Wangkeeree, $\mathrm{R}$, Boonking, U: A general iterative method for two maximal monotone operators and 2-generalized hybrid mappings in Hilbert space. Fixed Point Theory Appl. 2013, Article ID 246 (2013)

23. Rockafellar, RT: On the maximal monotonicity of subdifferential mappings. Pac. J. Math. 33, $209-216$ (1970)

24. Blum, E, Oettli, W: From optimization and variational inequalities to equilibrium problems. Math. Stud. 63, 123-146 (1994)

25. Combettes, PL, Hirstoaga, SA: Equilibrium programming in Hilbert spaces. J. Nonlinear Convex Anal. 6, 117-136 (2005)

26. Takahashi, S, Takahashi, W, Toyoda, M: Strong convergence theorems for maximal monotone operators with nonlinear mappings in Hilbert spaces. J. Optim. Theory Appl. 147, 27-41 (2010)

27. Censor, Y, Gibali, A, Reich, S: The split variational inequality problem. The Technion-Israel Institute of Technology, Haifa (2010). arXiv:1009.3780

10.1186/1687-1812-2014-20

Cite this article as: Yu et al.: Mathematical programming with multiple sets split monotone variational inclusion constraints. Fixed Point Theory and Applications 2014, 2014:20 